\title{
Recalibration in Validation Studies of Diabetes Risk Prediction Models: A Systematic Review
}

\author{
Katya L. Masconi ${ }^{1,2}$, Tandi E. Matsha ${ }^{3}$, Rajiv T. Erasmus ${ }^{1}$ and Andre P. Kengne ${ }^{2,4,{ }^{*}}$ \\ ${ }^{1}$ Division of Chemical Pathology, Faculty of Health Sciences, National Health Laboratory Service (NHLS) and \\ University of Stellenbosch, Cape Town, South Africa \\ ${ }^{2}$ Non-Communicable Diseases Research Unit, South African Medical Research Council, South Africa \\ ${ }^{3}$ Department of Biomedical Technology, Faculty of Health and Wellness Sciences, Cape Peninsula University \\ of Technology, Cape Town, South Africa
}

${ }^{4}$ Department of Medicine, University of Cape Town, Cape Town, South Africa

\begin{abstract}
Background: Poor performance of risk prediction models in a new setting is common. Recalibration methods aim to improve the prediction performance of a model in a validation population, however the extent of its application in the validation of diabetes risk prediction models is not yet known.

Methods: We critically reviewed published validation studies of diabetes prediction models, selected from five recent comprehensive systematic reviews and database searches. Common recalibration techniques applied were described and the extent to which recalibration and impacts were reported analysed.

Results: Of the 236 validations identified, $22.9 \%(n=54)$ undertook recalibration on existent models in the validation population. The publication of these studies was consistent from 2008. Only incident diabetes risk prediction models were validated, and the most commonly validated Framingham offspring simple clinical risk model was the most recalibrated of the models, in 4 studies $(7.4 \%)$.

Conclusions: This review highlights the lack of attempt by validation studies to improve the performance of the existent models in new settings. Model validation is a fruitless exercise if the model is not recalibrated or updated to allow for greater accuracy. This halts the possible implementation of an existent model into routine clinical care. The use of recalibration procedures should be encouraged in all validation studies, to correct for the anticipated drop in model performance.
\end{abstract}

Keywords: Risk prediction, diabetes, update, recalibration, validation.

\section{BACKGROUND}

The use of risk prediction models in a validation population is expected to have an effect on the performance of the model (usually a drop in the performance) due to the differences between development and validation populations, particularly the variances in outcome frequency between the populations, case-mix and measurements used for the variables and outcome determination [1]. In an effort to improve the performance of a model in a new setting, updating strategies have been proposed [2, 3]. The updating strategies range from simple adjustment of models' parameters to more complex model alterations. Simple updating methods, termed recalibration, describes the re-estimation of the model intercept (or baseline risk parameter) with or without reestimation of the regression coefficients.

The recalibration of risk prediction models is encouraged, where the resulting updated model

*Address correspondence to this author at the South African Medical Research Council, PO Box 19070, Tygerberg, 7505, Cape Town, South Africa; Tel: +27

21 9380841; Fax: +27 21 9380460; E-mail: andre.kengne@mrc.ac.za combines the prediction information that was captured in model development with the information of the new population. This lends to the concept that risk prediction models should be based on as many individuals' data as possible. Too often, existent models are externally validated and when performance is disappointing, a new prediction model is developed. This results in a large number of models available, which are all poorly externally validated [4]. For illustration, a systematic review by Noble and coworkers [5] found that between 1993 and 2011, over 145 models were developed to predict prevalent or incidents diabetes, of which only a few were externally validated. This is of concern, considering that use of accurate and validated risk models is increasingly advocated as a basis for risk screening in strategies to prevent the occurrence of diabetes among those at high risk, to promote early detection among those with prevalent undiagnosed diabetes, and tailoring the complexity and intensity of the management among those with diagnosed diabetes, to the risk of subsequent complications. Indeed, with diabetes mellitus growing to the epidemic proportions around the world, and considering the complexity of the interaction 
of factors contributing to diabetes occurrence and related complications, the ability of risk prediction models to incorporate a multitude of risk factors, accounting for this complexity, cements their importance in diabetes prevention and control strategies. Beyond the field of diabetes and noncommunicable diseases in general, with the opening era of personalised healthcare, prediction models will be increasingly used to assist clinical decision making. Efforts to limit the number of prediction models through careful updating of existing models to work in various settings, have a potential to improve their uptake in routine practice.

A recent validation study applied simple updating methods to diabetes risk prediction models, and reported some improvement, although non-optimal, of models performance [6]. However, the extent of the application of recalibration strategies in the validation of diabetes risk prediction models is not yet known. In this paper, we critically review the level of reporting, method of choice and extent of use of recalibration methods in validation studies, through a systematic review of studies on the validation of incident and prevalent diabetes risk prediction models, in an attempt to make conclusions on the extent of recalibration in diabetes risk prediction research.

\section{METHODOLOGY}

Building on the five most comprehensive review articles on both incident and prevalent diabetes risk prediction models by Buijsse et al. (2011) [7], Collins et al. (2011) [8], Noble et al. (2011) [5], Thoopputra et al. (2012) [9], and Brown et al. (2012) [10], additional relevant articles were identified through a systematic literature review according to the PRISMA guidelines, where necessary [11]. We searched PubMed for all published studies aimed at validating diabetes risk prediction models using the following string search: ((“diabetes" OR "diabetes mellitus" OR "type 2 diabetes") AND ("risk score" OR "prediction model" OR "predictive model" OR "predicting" OR "prediction rule" OR "risk assessment" OR "algorithm") AND ("validation" OR 'validate")).

Studies were included if they validated risk scores, models or questionnaires and the outcome was prevalent undiagnosed or incident diabetes in adults (aged $>18$ years). Studies undertaking internal validation were excluded as model recalibration should not be required at this early stage. Additionally, studies aimed at validating guidelines in new populations were excluded. Models that were developed outside of the logistic, cox or Weibull development methods were excluded due to the inability to validate these models (e.g. classification tree analysis method). There was no restriction on the variables included in the models, both non-invasive and invasive models were included. Additionally, there was no restriction on sample size or country. The data extracted included country/setting, name of the models validated, whether the study aimed at validation alone or with development of a model and the presence of a discussion and action (or lack thereof) on the recalibration of models. We reviewed the included studies with the aim of providing the reader with a comprehensive list of validated models, instances and prevalence of model recalibration, as well as the possible increase in performance of the updated model.

\section{RESULTS}

\section{Overview of Included Studies}

Following the sifting process, a total of 94 articles were included (Figure 1). These articles included 70 models and 236 validations were conducted. Figure 2 depicts the distributions of risk prediction model validation. Included published studies undertook the validation of existent diabetes risk prediction model/s, where validation refers to the process of evaluating the performance of a model. Studies were focussed on external validation which goes beyond the assessment of model performance in all or a portion of the developmental datasets by assessing the performance in an independent dataset. The validation of a model can be grouped by a hierarchy proposed by Justice et al. (1999) [12], according to the reproducibility and historic, geographic, methodologic, spectrum and follow-up period transportability (Text Box 1). Additionally, one paper can report on the validation of more than one model. Many studies undertook the validation of a model(s) as an added section to the development of a model in their population group $(48.8 \%)$. Details of the included studies are provided in Table 1; published between 1997 and 2014, but most appeared in 2005-2011. Articles reporting recalibration of existent models only appeared from 2008 onwards with the most appearing in 2010. The number and combination of predictors was variable, with age, sex, body mass index and waist circumference being the most commonly used variables. The study setting was highly heterogeneous; models were validated in 31 countries across 5 continents (only 1 from Africa). Models predicting incident diabetes were more 


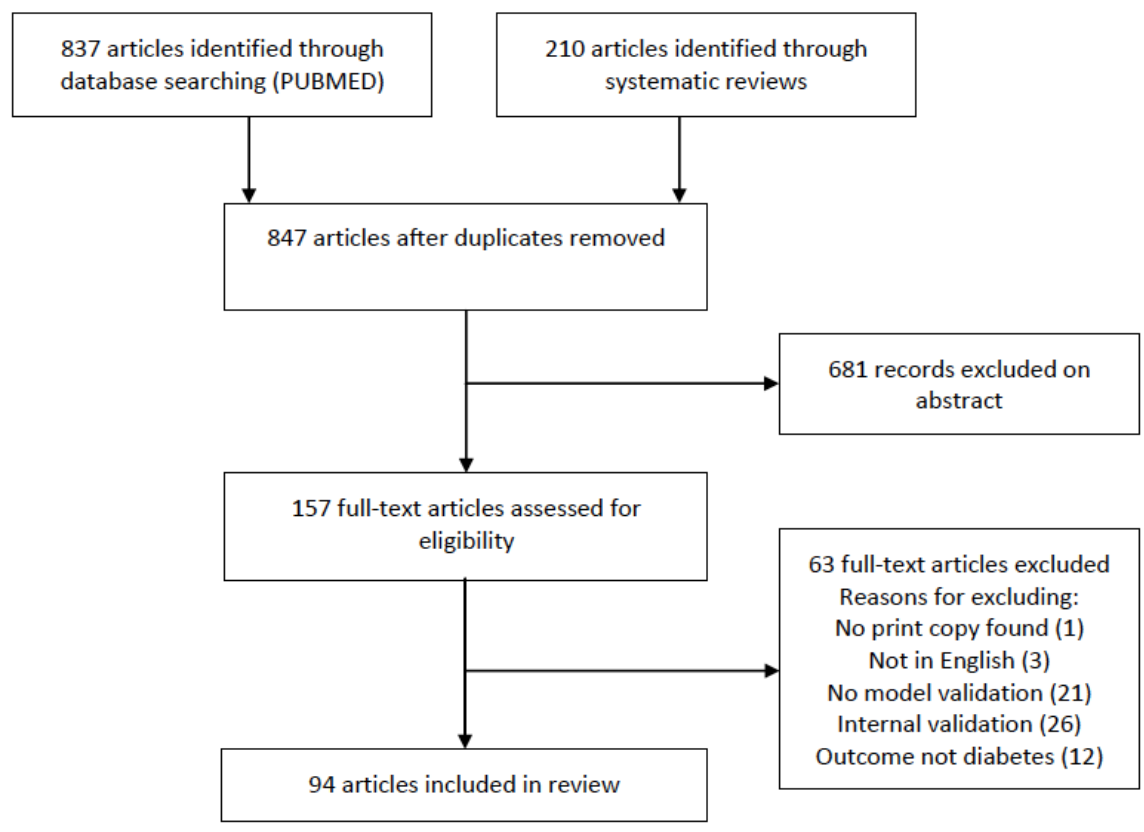

Figure 1: Flow diagram of selected studies.

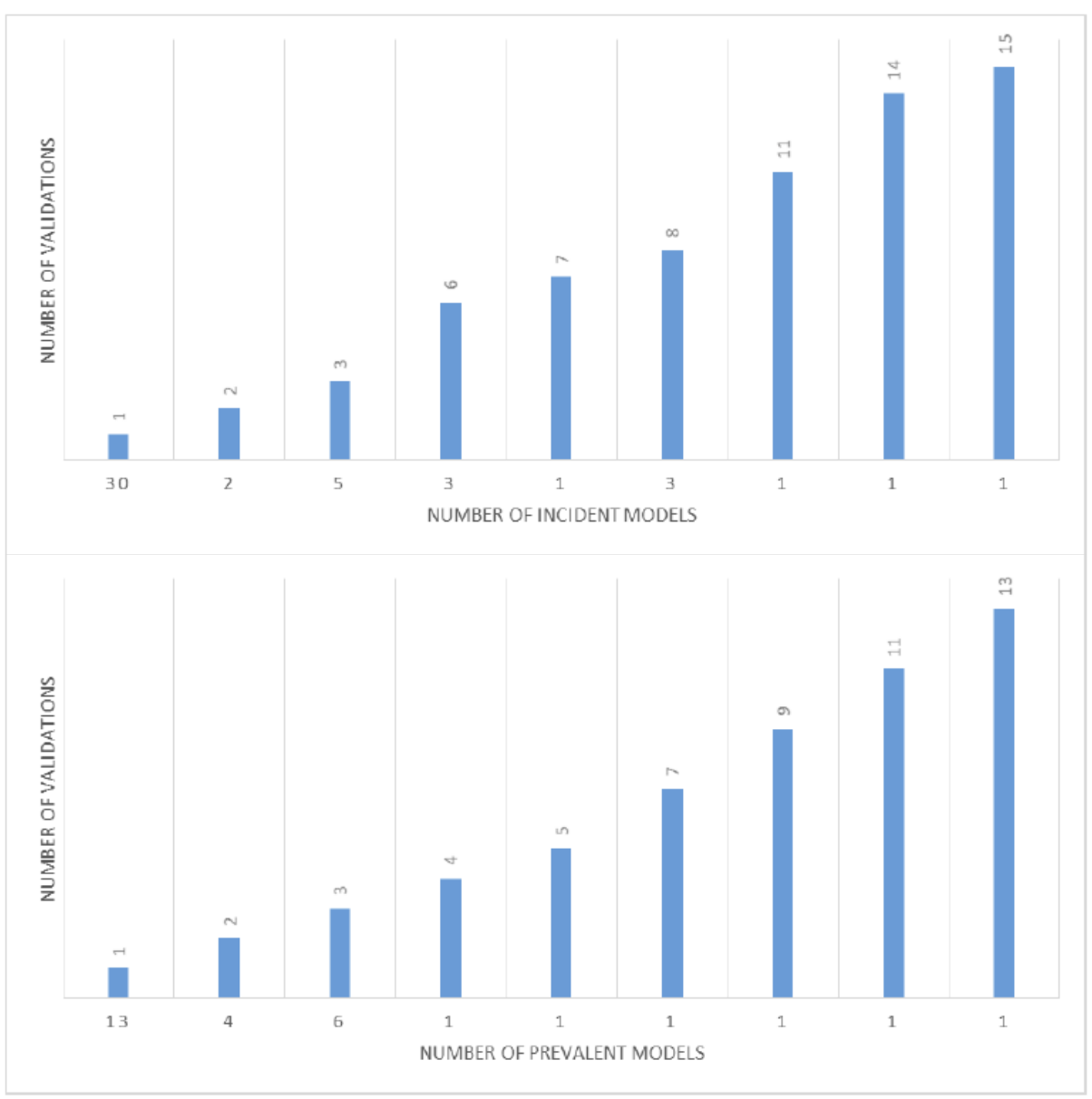

Figure 2: Bar diagram illustrating of frequency of incident (top) and prevalent (bottom) models validation.

The numbers on the X-axis indicate the number of models for each frequency, and the number at the tip of each bar indicate the frequency of model validation. The top 3 validated incident diabetes models are: 15 times - Framingham offspring simple clinical diabetes model; 14 times - San Antonio clinical risk model; 11 times - Cambridge diabetes risk score. The top 3 validated prevalent diabetes models: 13 times - Full prevalent FINDRISC risk model; 11 times - Rotterdam risk predicative model 1; 9 times - Cambridge diabetes risk model. 
Text box 1: A Hierarchy of External Validation of Predictive Systems - Adapted from Justice et al. [12]

\begin{tabular}{|c|c|}
\hline Level of validation & Cumulative generalizability evaluated \\
\hline \hline 0: Internal validation & Reproducibility \\
\hline 1: Prospective validation & $\begin{array}{c}\text { Level 0 + historic transportability } \\
\text { transportability }\end{array}$ \\
\hline 2: Independent validation & Level 2 at multiple sites \\
\hline 3: Multisite validation & Level 3 by multiple investigators \\
\hline 4: Multiple independent validation & Level 4 + follow-up period transportability \\
\hline
\end{tabular}

commonly validated $(62.7 \%)$ when compared to prevalent diabetes risk prediction. The development, recalibration and use of incident and prevalent risk prediction models vary and will therefore be discussed separately.

\section{Incident Diabetes Risk Prediction Models}

The most commonly validated model was the Framingham offspring simple clinical risk model [13] $(10.1 \%)$ followed by the San Antonio clinical risk model [14] (9.5\%). Validations were ranked according to the levels of transportability. There was no evidence of level 4 or 5 diabetes risk prediction validation. The most common form of validation (level 2) tested the models' geographic, methodologic and spectrum transportability in addition to the reproducibility and historic transportability $(62.8 \%)$. This included models which were validated in the same country as their development but a different city or cohort to development, as well as validation of a model for a different outcome.

\section{Prevalent Diabetes Risk Prediction Models}

The Finnish diabetes full risk model was the most frequently validated prevalent diabetes risk prediction model $(14.8 \%)$ [15, 16], followed by the Rotterdam predictive model 1 (12.5\%). As with incident risk models, hierarchy level 2 was the most common level of validation ( $81.8 \%)$, with no level 4 or 5 validation.

\section{Recalibration Methods}

Multiple updating methods exist [2, 3, 17-19], varying in the complexity and the number of parameters that are adjusted or re-estimated. The term 'recalibration' is used to describe basic techniques to fit a predictive model to a new setting. The development of the model dictates the recalibration methods available. The mathematical model chosen for development may follow logistic regression, cox or Weibull principles. The intercept, or equivalent, of risk models is determined by the prevalence of the outcome in the population in which the model was developed and the updating of this intercept aims to solve the discrepancy between the mean predicted risk and mean observed risk resulting in better calibration. To be noted, recalibration, through either method, does not change the discriminatory ability of the risk prediction model as the relative ranking of the predicted probabilities remain the same [20].

Logistic regressions are the most commonly used for risk prediction research. Recalibration methods, described by Steyerberg [1] and Janssen et al. [3], aim to update the intercept of logistic models to better account for the prevalence in the validation population. The intercept can be updated by fitting a logistic regression model with a linear predictor as the only covariate in the updating set or by calculating a correction factor that is based on the mean predicted risk and observed outcome frequency in the validation population. When the outcome frequency is not particularly low or high, the correction factor will equal the calibration intercept. The final correction factor is simply added to the intercept of the original model. This is considered the most basic form of logistic model updating. An additional method, termed logistic calibration, fits a logistic regression model with a linear predictor as the only covariate in the updating set [3]. The calibration slope is used to recalibrate (multiply by) the original regression coefficients. The closer the calibration slope is to 1 , the less adjustment the original regression coefficients required. The intercept is also updated by adding the calibration intercept to the intercept of the original prediction model.

Survival models available for risk prediction research depend on the distribution assumptions that can be made. Weibull models are generalised 


\begin{tabular}{|c|c|c|c|c|c|c|c|c|c|c|c|c|c|c|c|}
\hline 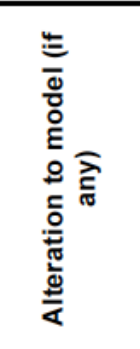 & 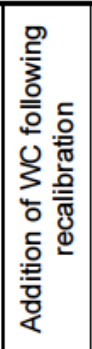 & 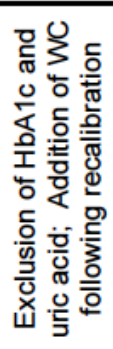 & 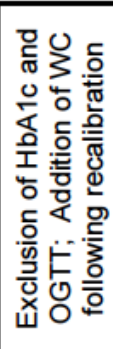 & & & & & & & 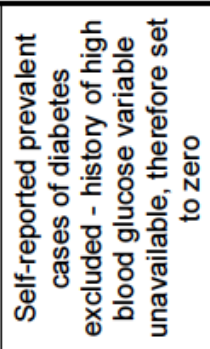 & & & & & 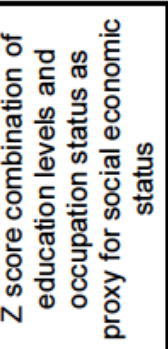 \\
\hline 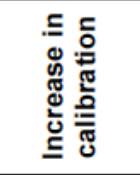 & 우 & $\stackrel{\infty}{>}$ & $\stackrel{\mathscr{D}}{\nu}$ & $\stackrel{\infty}{\sim}$ & & & & & & & & & & & \\
\hline 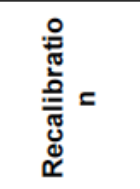 & \multicolumn{3}{|c|}{$\stackrel{\Perp}{\nu}$} & 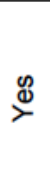 & & & & & & & & & & & \\
\hline 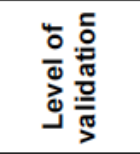 & \multicolumn{3}{|c|}{$N$} & $m$ & & & & & & & & & & & \\
\hline - & \multicolumn{3}{|c|}{ 을 } & zo & & & & & & & & & & & \\
\hline 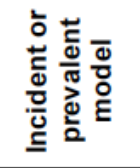 & \multicolumn{3}{|c|}{ 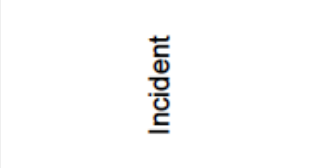 } & \multicolumn{12}{|l|}{ 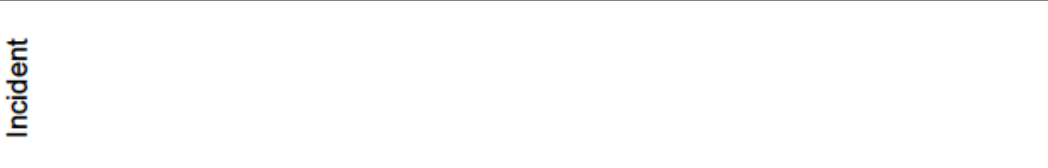 } \\
\hline 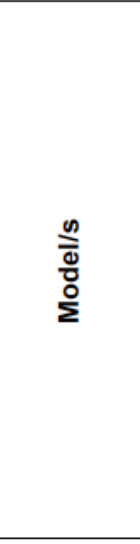 & 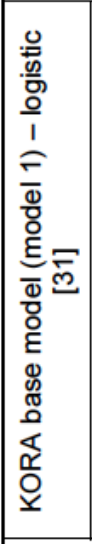 & 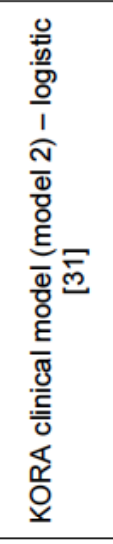 & 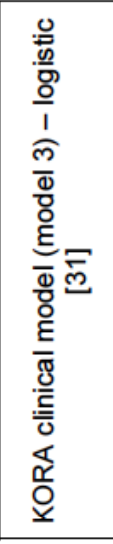 & 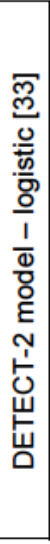 & 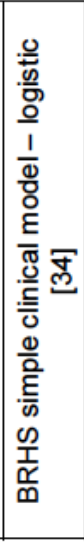 & 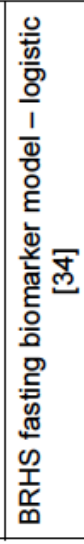 & 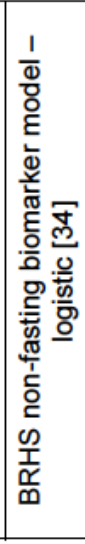 & 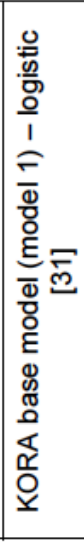 & 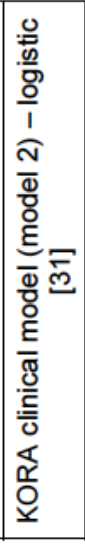 & 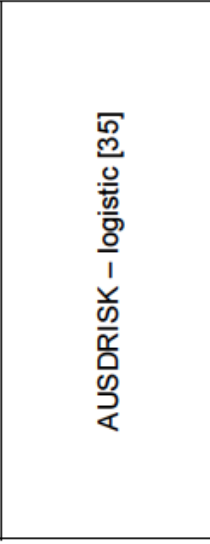 & 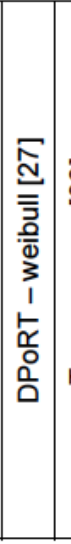 & 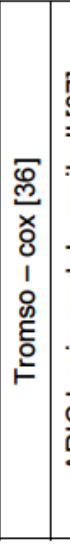 & 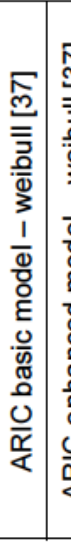 & 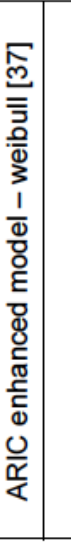 & 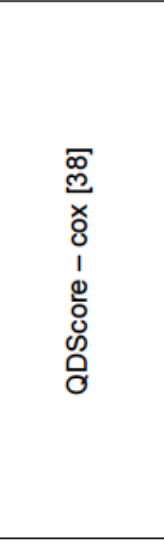 \\
\hline 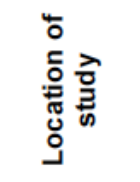 & \multicolumn{3}{|c|}{ 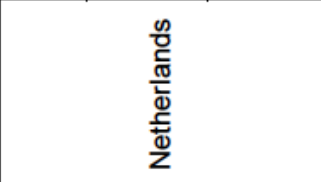 } & \multicolumn{12}{|l|}{ 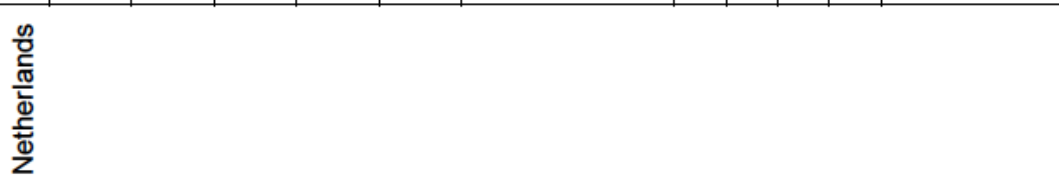 } \\
\hline 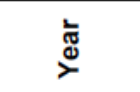 & \multicolumn{3}{|c|}{$\stackrel{\sim}{\sim}$} & \multicolumn{12}{|l|}{$\stackrel{\sim}{\sim}$} \\
\hline 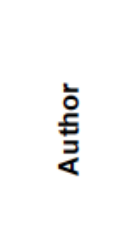 & \multicolumn{3}{|c|}{$\begin{array}{l}\frac{\bar{p}}{\frac{m}{\sigma}} \\
\frac{\pi}{\Phi} \\
\bar{w} \\
\frac{0}{0} \\
\frac{0}{\alpha}\end{array}$} & \multicolumn{12}{|l|}{ 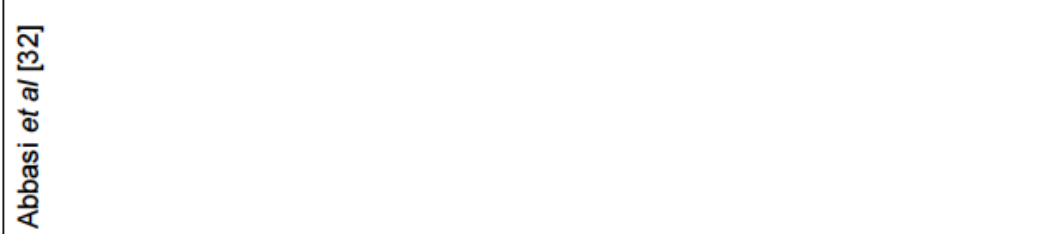 } \\
\hline
\end{tabular}




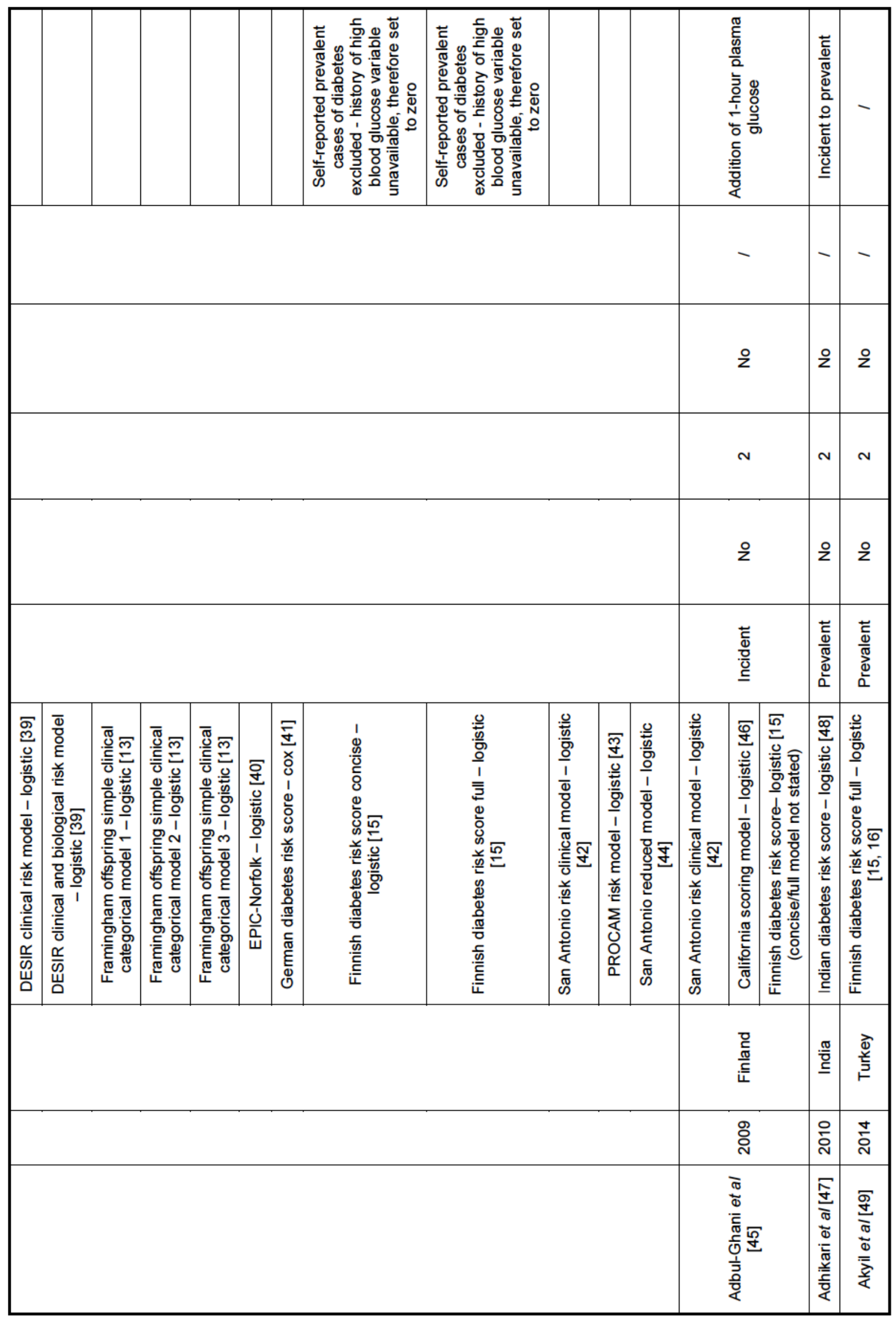




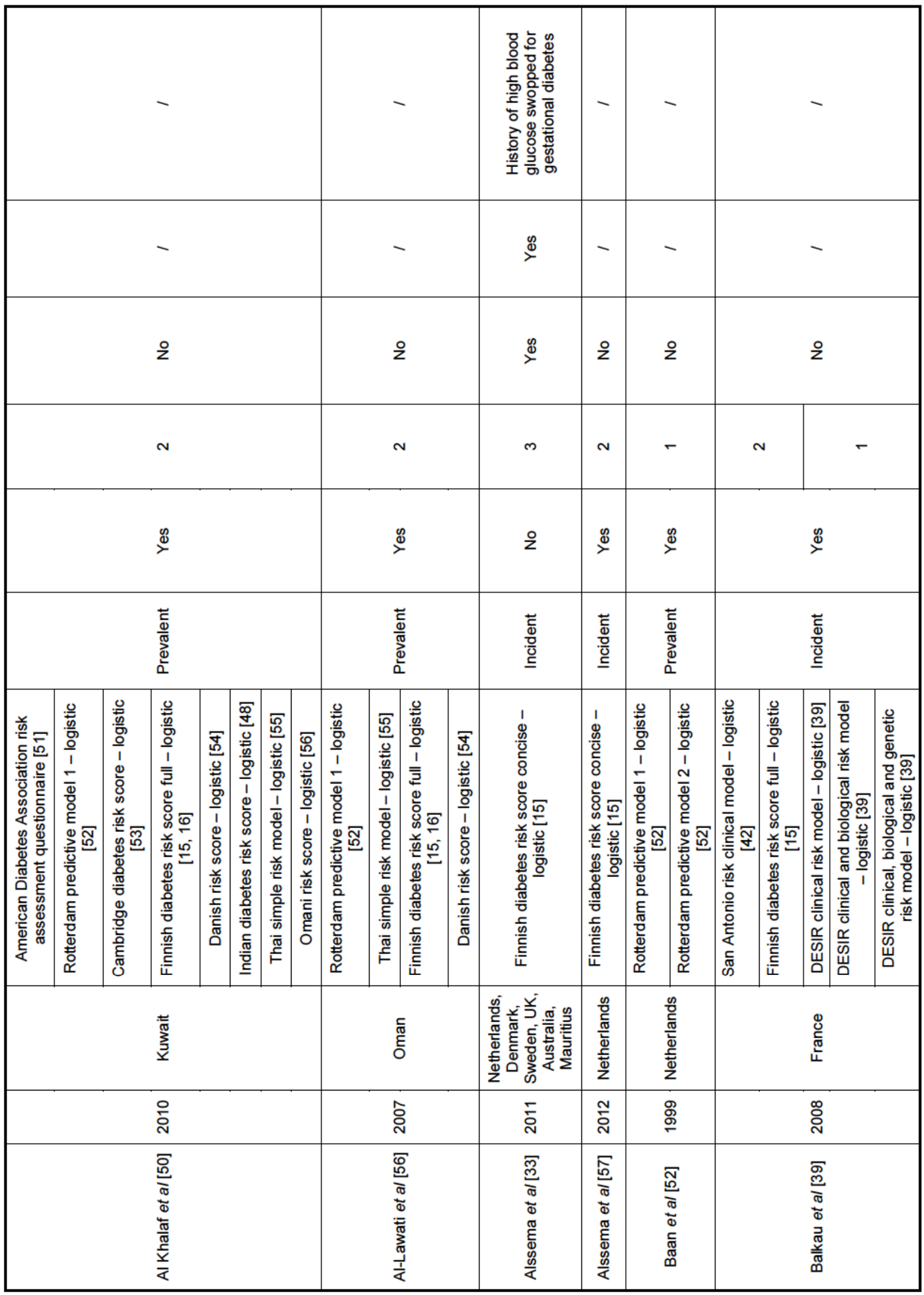




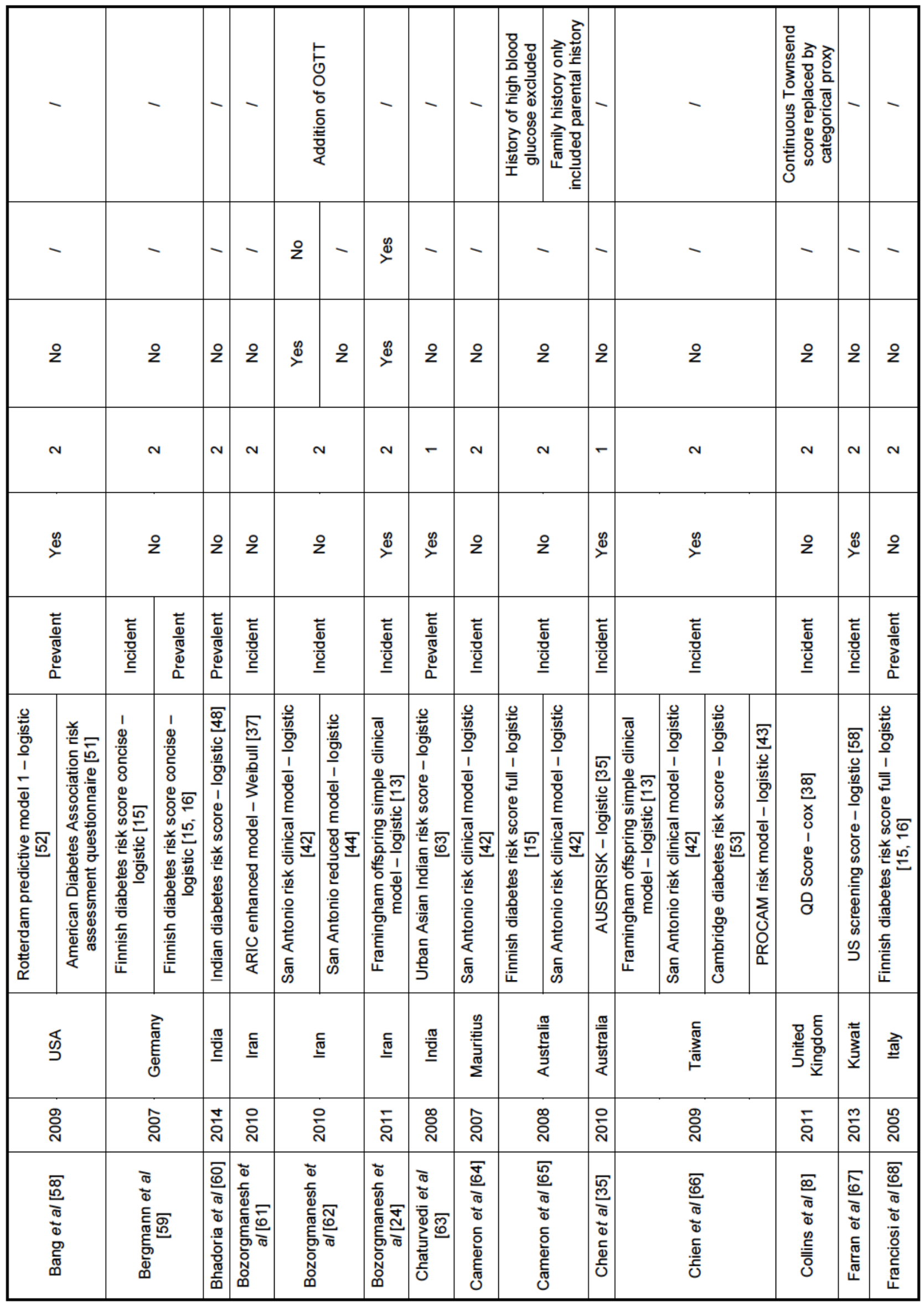




\begin{tabular}{|c|c|c|c|c|c|c|c|c|c|c|c|c|c|c|c|c|c|c|c|c|}
\hline & & & - & & & & & - & - & 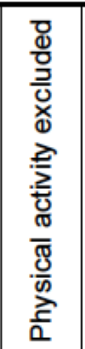 & - & - & - & - & - & - & - & & - & - \\
\hline & & & - & & & & & - & - & - & - & - & - & - & - & - & - & & - & - \\
\hline & & & 운 & & & & & $\stackrel{\circ}{z}$ & zo & 은 & $\stackrel{\circ}{z}$ & 오 & 은 & $\frac{c}{2}$ & 운 & 을 & 우 & & 울 & 을 \\
\hline- & & & $c$ & & & & & N & - & $N$ & $m$ & $r$ & - & $\Omega$ & N & $r$ & - & & - & $N$ \\
\hline & & & $\stackrel{\mathscr{\infty}}{\succ}$ & & & & & $\stackrel{\circ}{z}$ & $\stackrel{\mathscr{\infty}}{\circlearrowright}$ & 은 & 우 & $\stackrel{\infty}{\rightleftharpoons}$ & $\stackrel{\infty}{\nu}$ & $\frac{c}{2}$ & zo & $\stackrel{\infty}{\nu}$ & $\stackrel{\infty}{\check{\nu}}$ & & $\stackrel{\infty}{>}$ & $\stackrel{\infty}{\rightleftharpoons}$ \\
\hline & & & 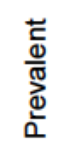 & & & & & 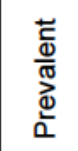 & 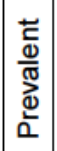 & 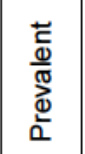 & 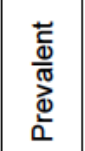 & 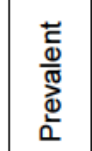 & 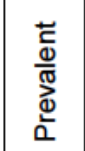 & $\frac{1}{d}$ & 螜 & 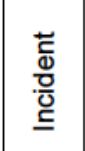 & 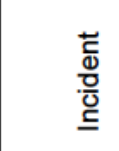 & & 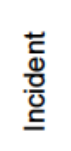 & 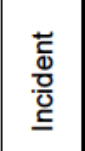 \\
\hline 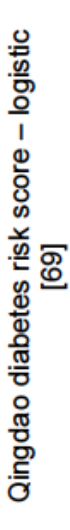 & 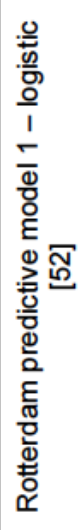 & 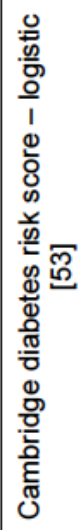 & 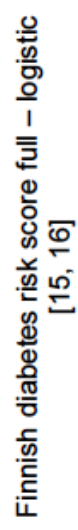 & 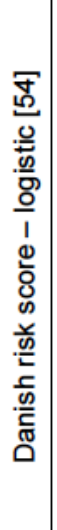 & 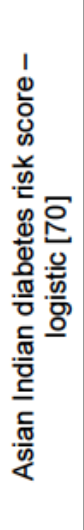 & 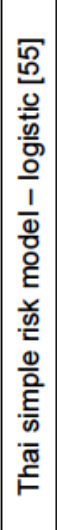 & 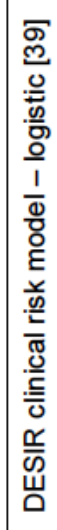 & 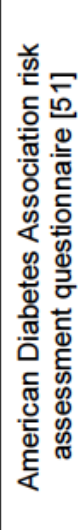 & 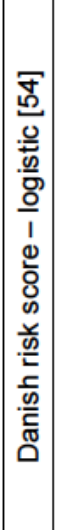 & 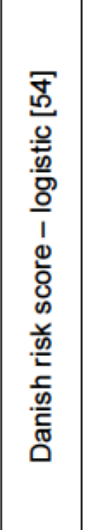 & 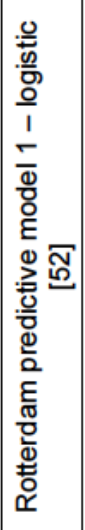 & 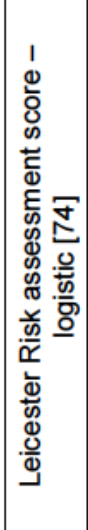 & 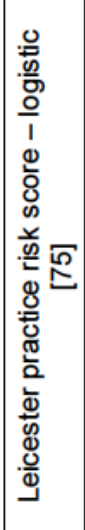 & 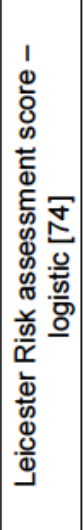 & 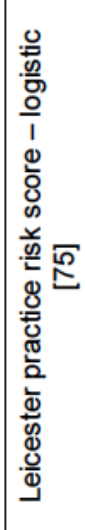 & 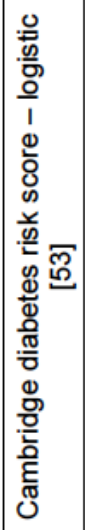 & 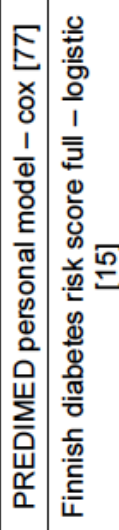 & 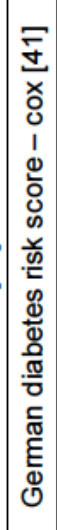 & 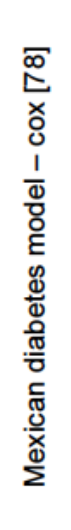 & 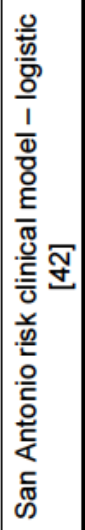 \\
\hline & & & 胥 & & & & & હ & 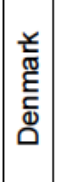 & 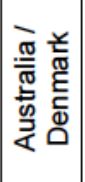 & $\begin{array}{l}\bar{\pi} \\
\text { 응 } \\
\text { 는 }\end{array}$ & 弚 & 弚 & 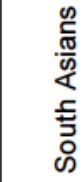 & 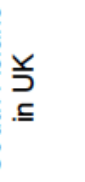 & 弚 & $\begin{array}{l}\frac{5}{\pi} \\
\text { की }\end{array}$ & & $\begin{array}{l}\stackrel{\circ}{x} \\
\frac{\mathbb{x}}{\Sigma}\end{array}$ & ભ \\
\hline & & & 웅 & & & & & ڤ્ণ & ठ্ণ & 足 & ષั & 울 & $\stackrel{\sim}{\sim}$ & 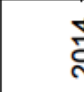 & & ঃి & $\stackrel{\sim}{\sim}$ & & 울 & ర્ત \\
\hline & & & 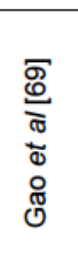 & & & & & 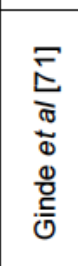 & 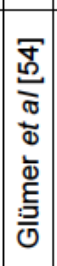 & 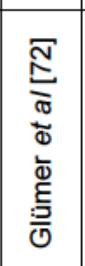 & 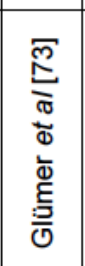 & $\begin{array}{l}\frac{F}{N} \\
\frac{\pi}{\pi} \\
\frac{\pi}{0} \\
\frac{\pi}{0} \\
\frac{0}{0}\end{array}$ & 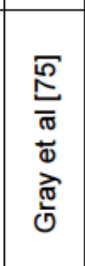 & (c) & $\frac{\sqrt{2}}{\frac{2}{\pi}}$ & 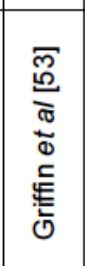 & 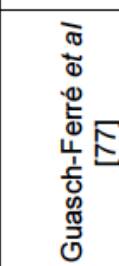 & & 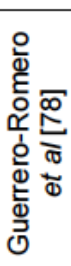 & 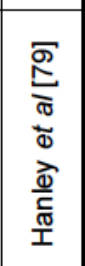 \\
\hline
\end{tabular}




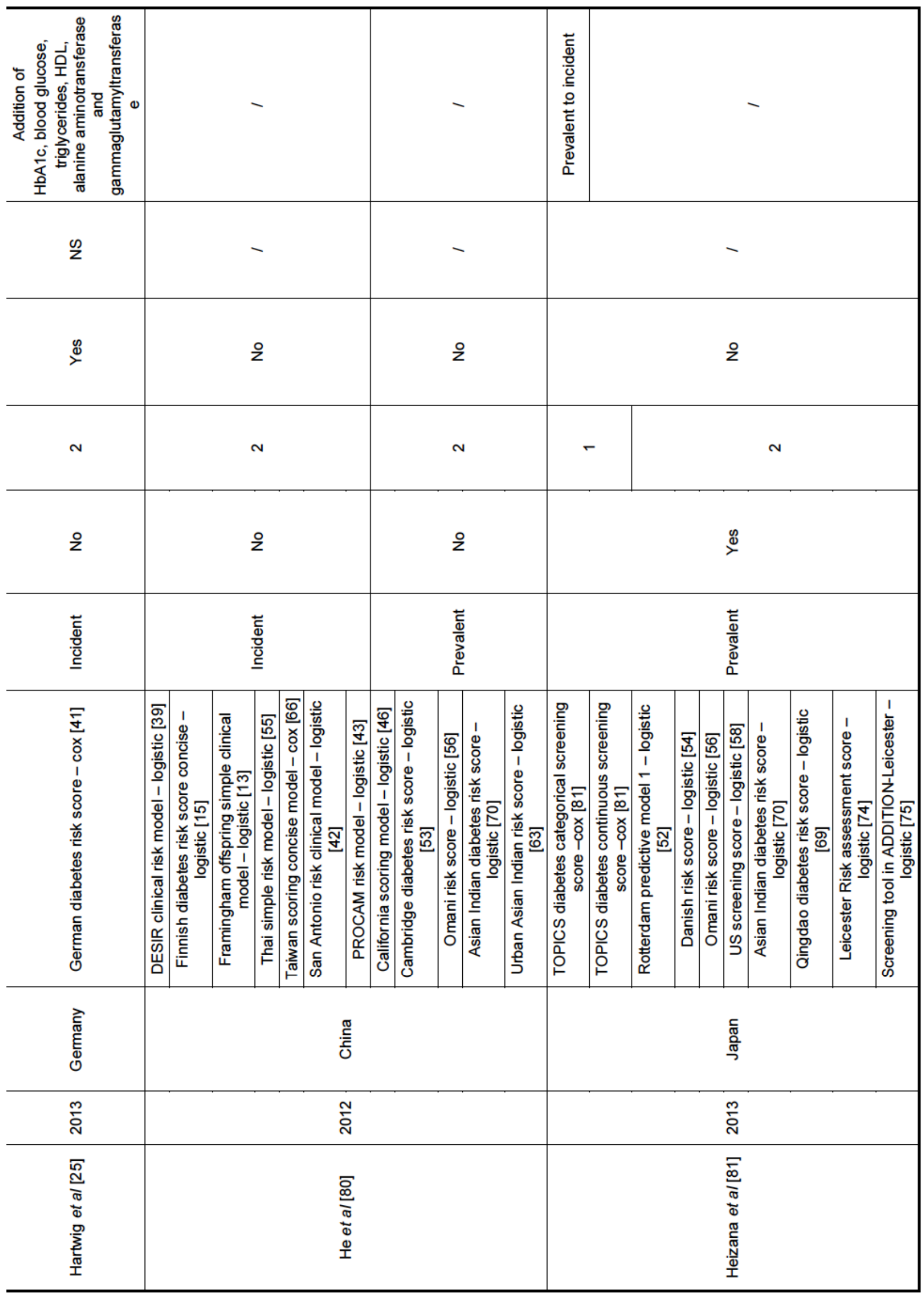




\begin{tabular}{|c|c|c|c|c|c|c|c|c|c|c|c|c|c|c|c|}
\hline- & - & - & - & & - & - & & & 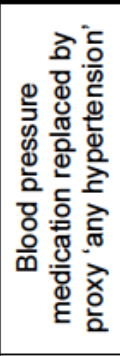 & 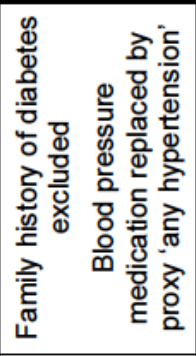 & & & 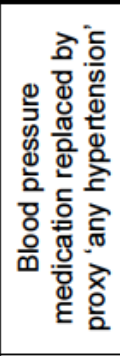 & 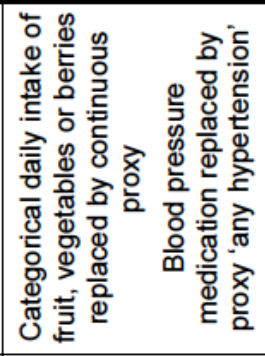 & \\
\hline- & - & N & - & & - & - & 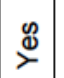 & 은 & $\stackrel{\infty}{\succ}$ & 운 & $\stackrel{\infty}{\rightleftharpoons}$ & ì & $\stackrel{\infty}{\succ}$ & $\stackrel{\infty}{>}$ & $\stackrel{\infty}{\rightleftharpoons}$ \\
\hline 운 & 울 & $\stackrel{\infty}{\nu}$ & 은 & & 울 & 울 & $\stackrel{\infty}{\succ}$ & & & & & & & & \\
\hline$N$ & $-N$ & $r$ & N & & - & - & $m$ & & & & & & & & \\
\hline$\stackrel{\circ}{z}$ & $\stackrel{\infty}{\nu}$ & 우 & $\stackrel{\mathscr{d}}{\nu}$ & & $\stackrel{\infty}{\nu}$ & $\stackrel{\Perp}{\nu}$ & 울 & & & & & & & & \\
\hline 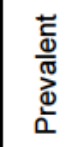 & 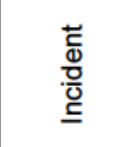 & 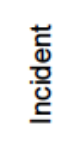 & 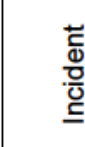 & & 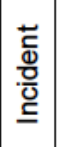 & 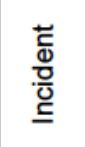 & 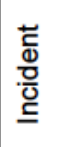 & & & & & & & & \\
\hline 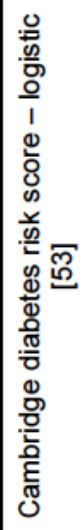 & 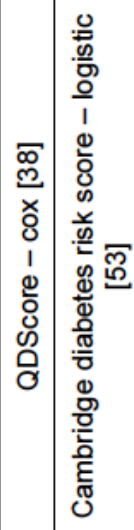 & 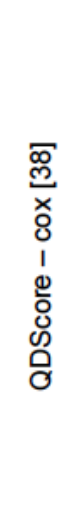 & 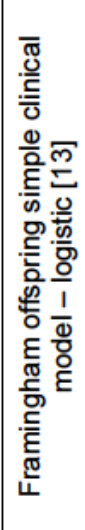 & 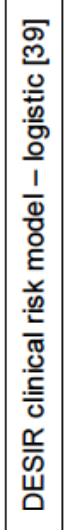 & 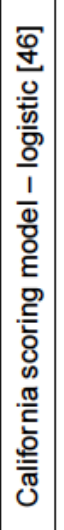 & 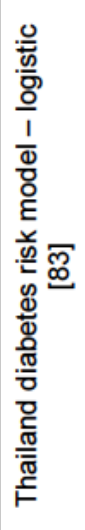 & 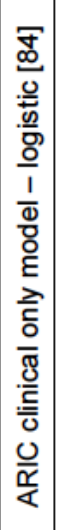 & 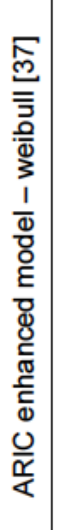 & 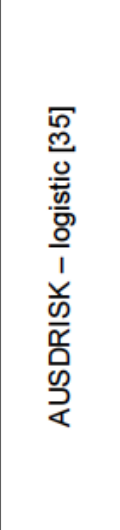 & 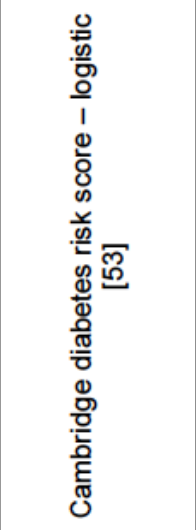 & 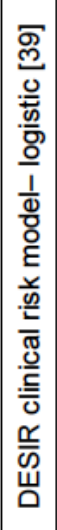 & 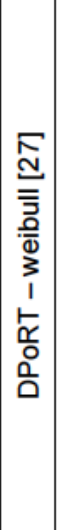 & 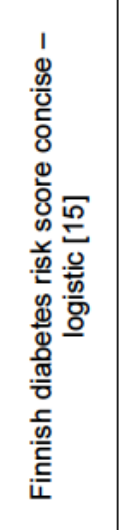 & 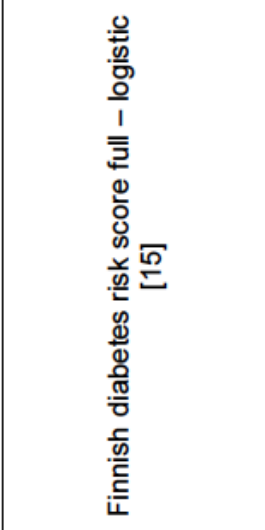 & 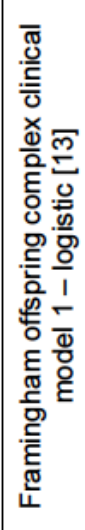 \\
\hline 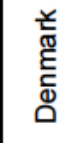 & 弚 & 弚 & 心 & & 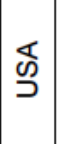 & 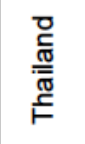 & 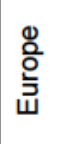 & & & & & & & & \\
\hline ¿্ర & 오 & $\stackrel{+}{\stackrel{N}{*}}$ & 올 & & ֻัర & ڤ్ & ָे & & & & & & & & \\
\hline 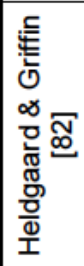 & 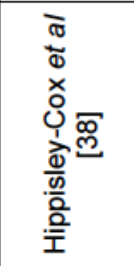 & 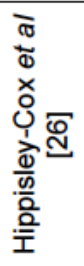 & 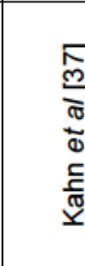 & & 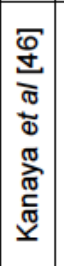 & 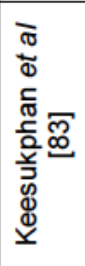 & 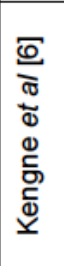 & & & & & & & & \\
\hline
\end{tabular}




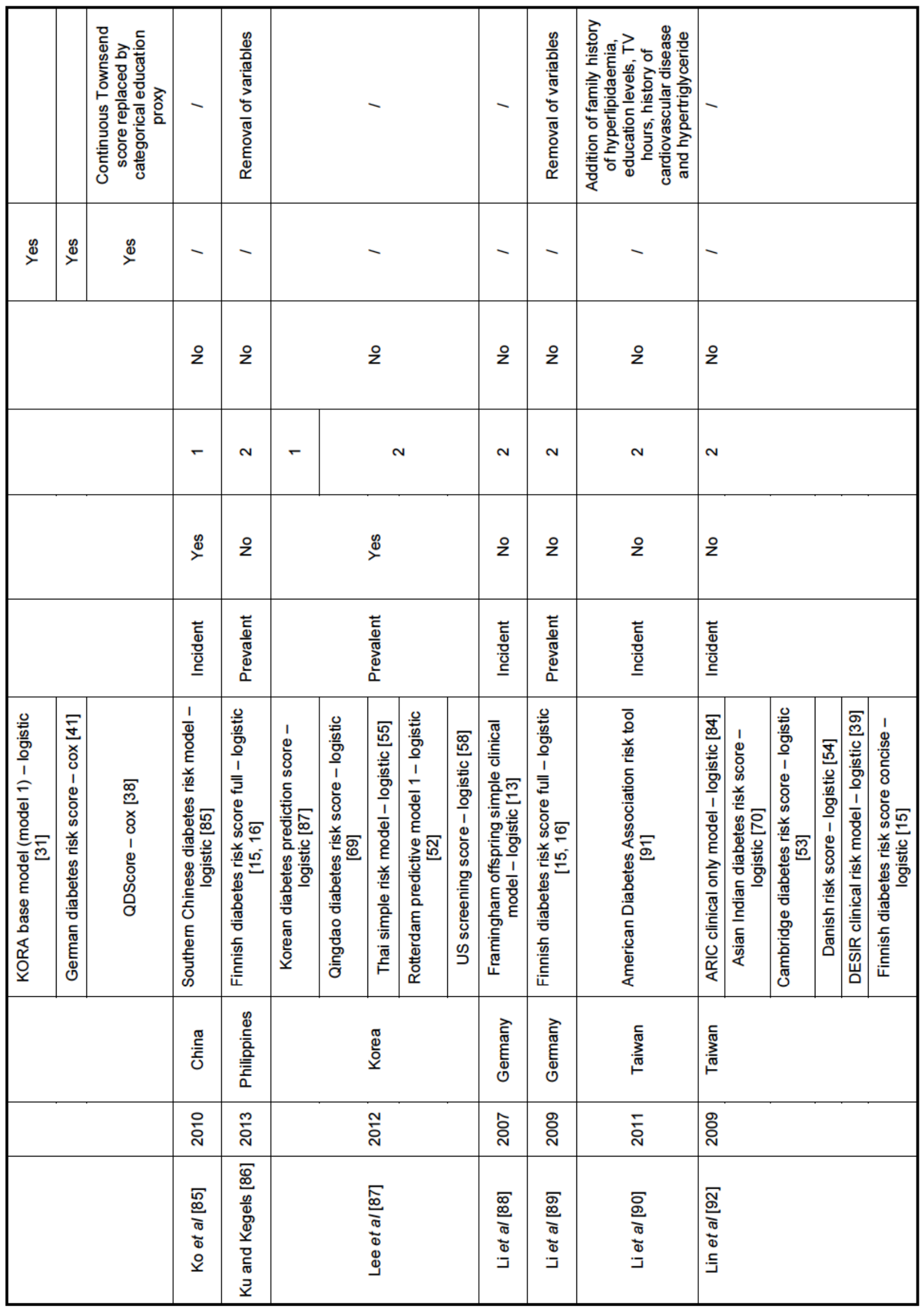




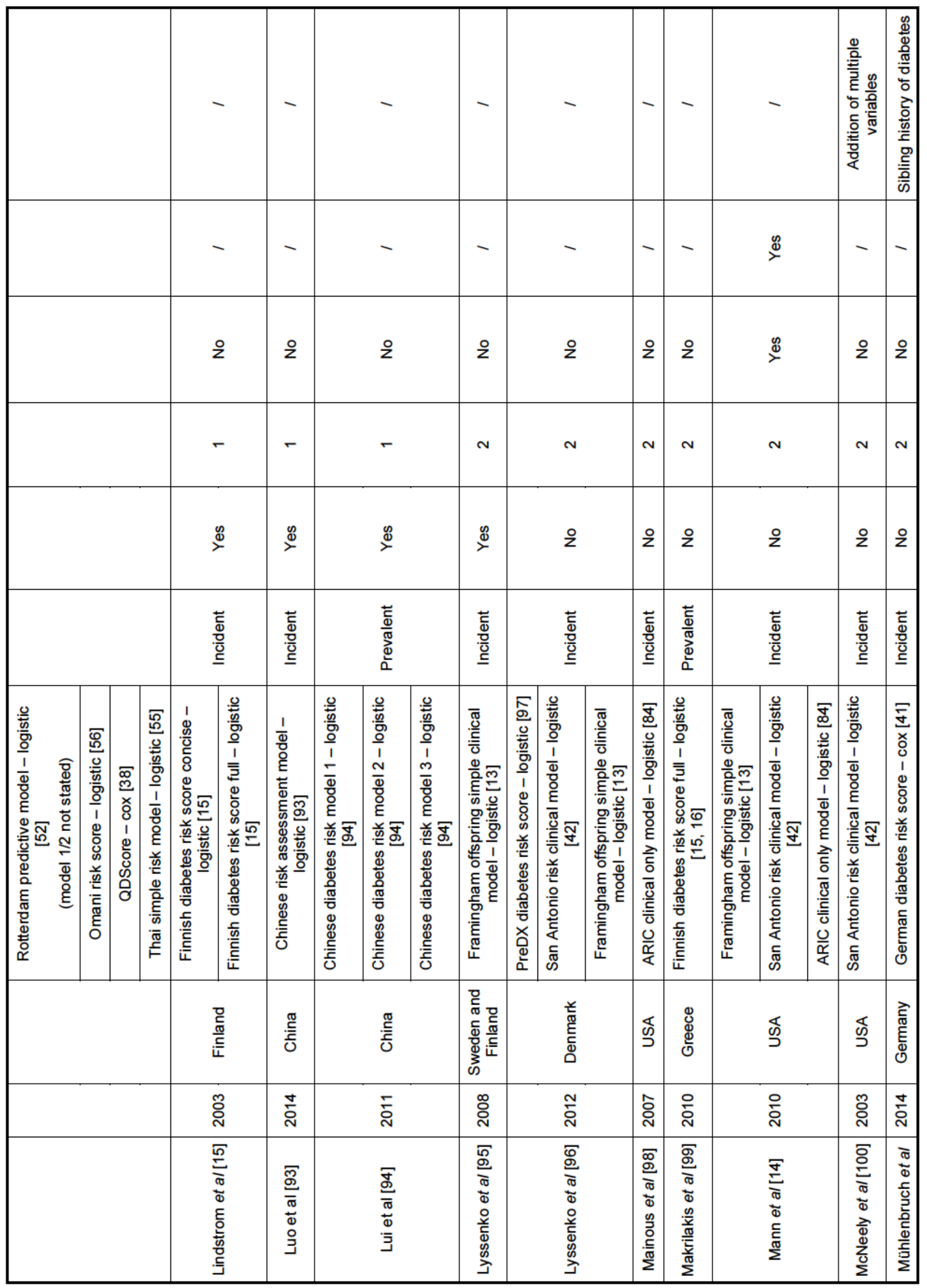




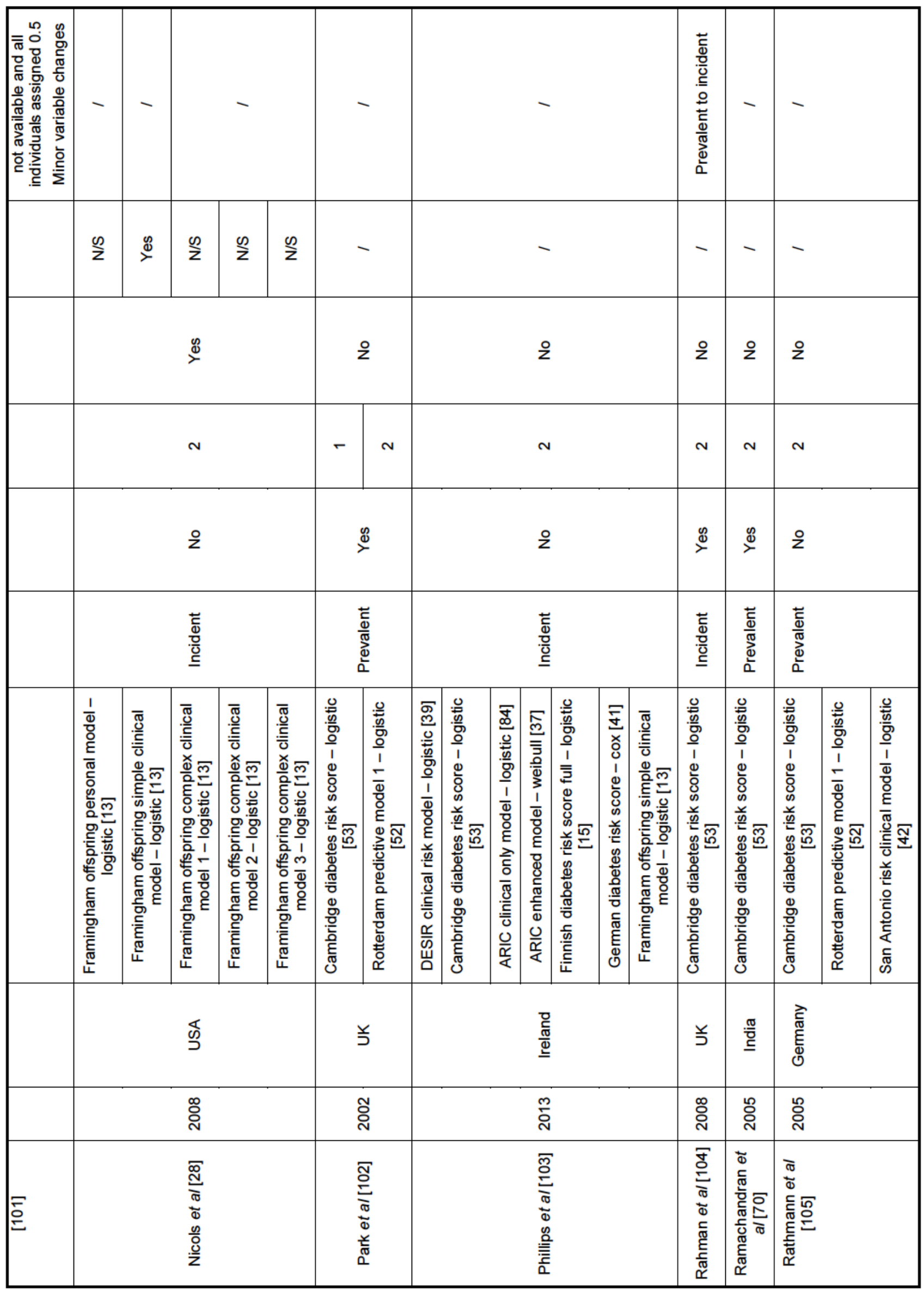




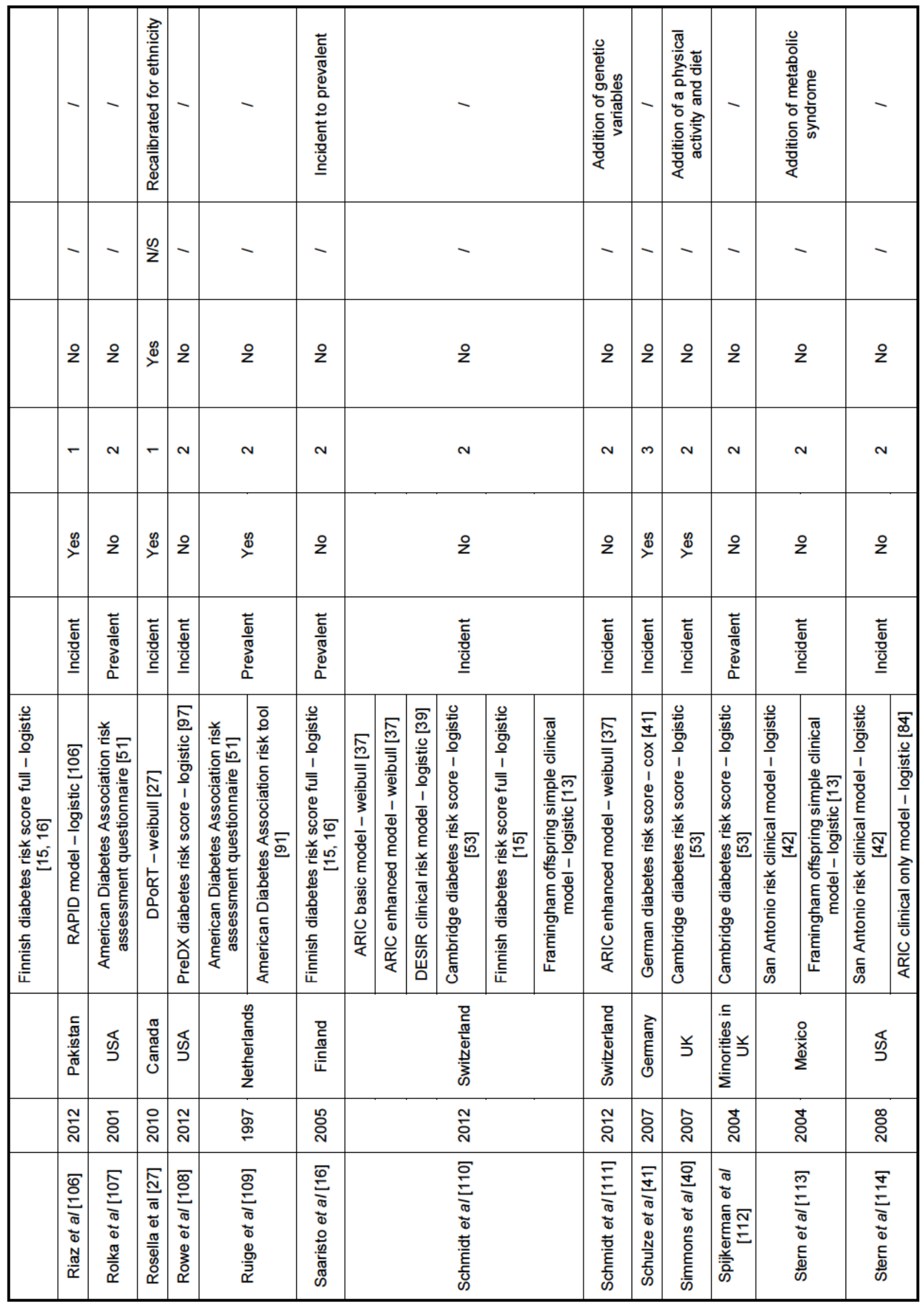




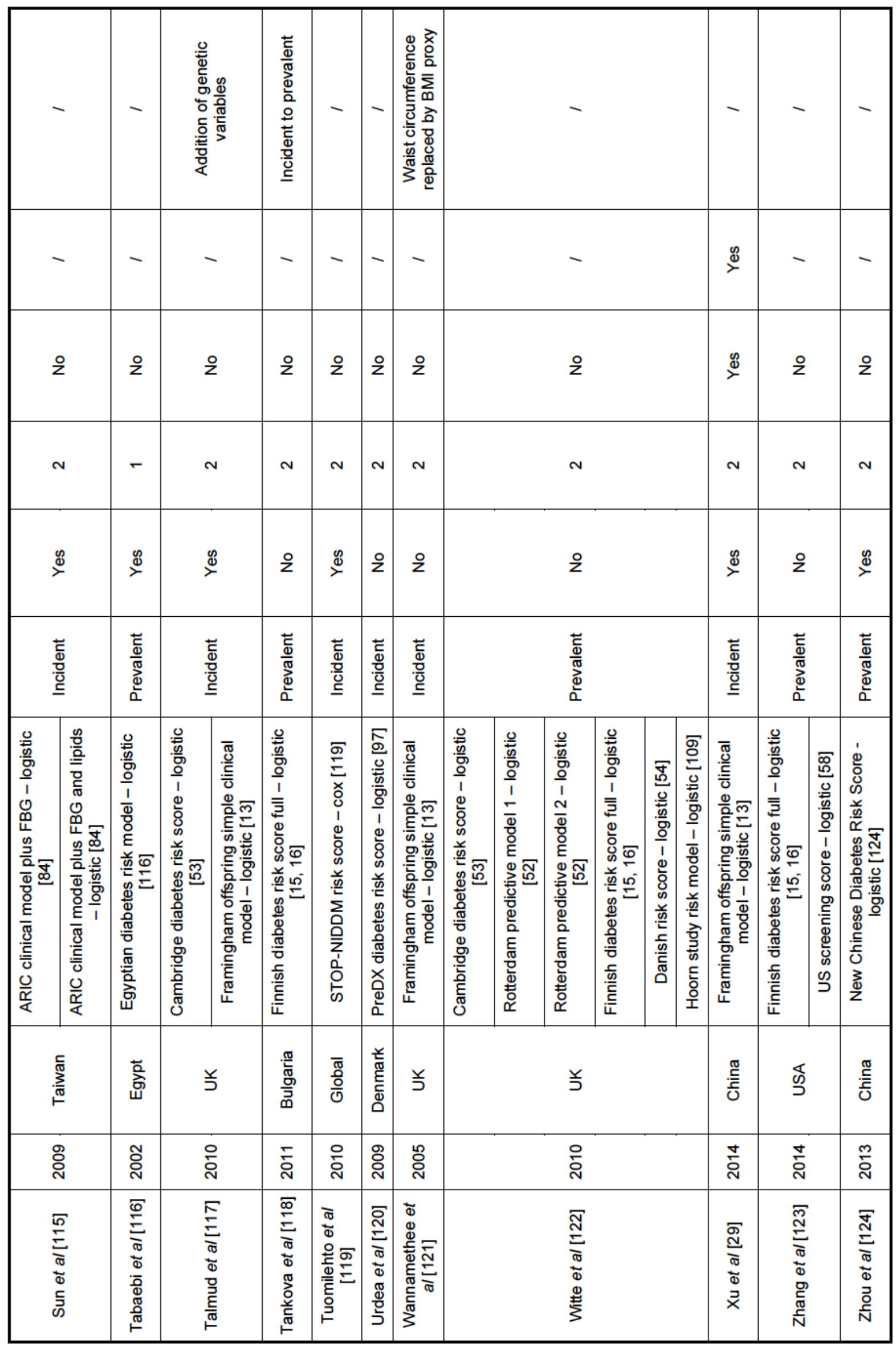


exponential models with the inclusion of shape (survival rate), allowing for more flexibility on the types of data that the model can fit. The model has a hazard function which measures how likely the outcome/event will take place as a function of the length of observation [21]. While exponential distribution has a constant hazard function, the Weibull distribution hazard rate can increase or decrease in relation to time. The Weibull model is a popular method for parametric data. When distribution assumptions of the survival time (time until diagnosis) cannot be met, the Cox proportional hazard model can be used. Additionally, cox models are used when the risk factors have a multiplicative effect on the hazard function and can be extended for multiple regression situations [22]. Cox models do not have an intercept but rather an equivalent, the 'baseline survival function' or 'baseline risk'. This baseline information is almost never given by authors of published medical articles that report a cox model, however it can be recalculated [23]. Cox models are often referred to as semi-parametric, as the baseline hazard function is non-parametric, while the linear predictor in the cox model is fully parametric.

The incorporation of diagnosis time in both of these models allows for them only to be used for the development of incident diabetes risk prediction models (as opposed to prevalent diabetes prediction). The choice of model is researcher dependent and each come with their own advantages, parametric models are more precise with smaller standard errors, while it is easier, and can prevent biases, not having to make assumptions of the underlying hazard function nature or shape with semi-parametric models. The recalibration of all survival analysis models use KaplanMeier to determine the average incidence rates and update the model to the validation population incidence rate [17]. Additionally available, the mean values of each variable within the model which were derived from the validation population is replaced by the mean values of the same variables from the validation population. These methods are described in more detail by D' Agostino (2001) [17]. Text box 2 details the components of the various models that are altered during recalibration.

\section{Reporting of Recalibration}

Of the 236 validations of diabetes risk prediction models in alternate populations, $54(22.9 \%)$ reported the use of recalibration methods in an effort to increase performance of the existent models. The reporting of the recalibration method was clear, the only article to not report the method of recalibration was Bozorgmanesh et al. (2011) [24]. 42 of these studies $(77.8 \%)$ reported an increase in model performance following the recalibration of the original model (seven studies did not report the original or recalibrated model performance [25-28]). Every recalibration was carried out on an incident diabetes risk prediction model, with most of them being logistic regression models $(75.9 \%)$. Additionally, $68.5 \%$ of recalibrations were carried out in level 3 calibrations. There was no one model that was recalibrated significantly more often than others. The Framingham offspring simple clinical model was recalibrated four times $(7.4 \%)[14,24,28,29]$, while the DPoRT, concise Finnish, German, KORA base, KORA clinical, QDScore and San Antonio clinical diabetes risk models were recalibrated three times $(5.6 \%)$.

\section{DISCUSSION}

The validation of existent models in a new population is highly encouraged, preventing the

Text box 2: Mathematical formula for key models illustrating change before and after recalibration - adapted from Janssen et al. [3] and Houwelingen [23]

\begin{tabular}{|c|c|c|c|}
\hline Model & Formula & Components & Recalibration change \\
\hline \multirow[t]{2}{*}{$\overline{\text { Logistic }}$} & \multirow{2}{*}{$\begin{array}{c}1 /\left\{1+\text { EXP }\left[-\left(ß_{0}+ß_{1} \times \text { predictor }_{1}\right.\right.\right. \\
\left.\left.\left.+\ldots+ß n \times \text { predictor }_{n}\right)\right]\right\}\end{array}$} & \multirow{2}{*}{$\begin{array}{c}\text { Intercept: } ß_{0} \\
\text { Variable coefficient: } ß_{1}-ß n\end{array}$} & Update intercept: $\beta_{0}+$ correction factor \\
\hline & & & $\begin{array}{l}\text { Update intercept: } \beta_{0}+\text { correction factor } \\
\text { Coefficient: linear predictor } \times \beta_{\text {calibration }}\end{array}$ \\
\hline \multirow[t]{2}{*}{ Cox } & \multirow{2}{*}{$\begin{array}{c}H_{o}(t) \operatorname{EXP}(x \beta) \text { where } \\
x \beta=\beta_{1}\left(x_{1}-M_{1+\ldots}+\beta_{n}\left(x_{n}-M_{n}\right)\right.\end{array}$} & \multirow{2}{*}{$\begin{array}{l}\text { Baseline hazard function: } \mathrm{H}_{0}(\mathrm{t}) \\
\text { Prognostic index: } x \beta \\
\text { Regression coefficient: } \beta \\
\text { Mean of risk factor: } M\end{array}$} & Update incidence rate of validation cohort: $\mathrm{H}_{0}(\mathrm{t})$ \\
\hline & & & Update mean value of variable in validation cohort: $\beta$ \\
\hline \multirow[t]{2}{*}{ Weibull } & \multirow[t]{2}{*}{$\begin{array}{c}\left(\beta_{0}+\beta_{1} \ln (t)\right) \operatorname{EXP}(x \beta) \text { where } \\
x \beta=\beta_{1}\left(x_{1}-M_{1}+\ldots+\beta_{n}\left(x_{n}-M_{n}\right)\right.\end{array}$} & \multirow{2}{*}{$\begin{array}{l}\text { Hazard function: } \beta_{0}+\beta_{1} \ln (t) \\
\text { Prognostic index: } x \beta \\
\text { Regression coefficient: } \beta \\
\text { Mean of risk factor: } M\end{array}$} & $\begin{array}{l}\text { Update incidence rate of validation cohort: } \beta_{0} \text { of }\left(\beta_{0}+\right. \\
\left.\qquad \beta_{1} \ln (t)\right)\end{array}$ \\
\hline & & & Update mean value of variable in validation cohort: $\beta$ \\
\hline
\end{tabular}


availability of numerous models, where few have been externally validated. The common method of developing and validating models simultaneously in a database in which previous risk prediction research has not been, defeats this purpose. Ideally, should a database suitable for diabetes risk prediction research be available, models should first be validated in an attempt to find an existent model that can perform at an optimum discrimination and calibration. Should a model show systematic overestimation or underestimation of risk, and the performance be too low to allow for accurate prediction and successful implementation, recalibration techniques can be employed in an effort to increase the performance of the model.

The aim of this study was to determine the extent to which model recalibration was undertaken in validation of diabetes risk models. This review of available published literature on the validation of diabetes risk prediction models showed that although validation of existent models is occurring, the attempt to fit these models to the new setting is poor. Additionally, we wished to determine if this recalibration was successful in increasing model performance when incorporating information for the validation population. Most studies that undertook the recalibration of models were able to show that model performance can be increased with basic recalibration techniques. The new models retain their importance in a new setting, taking into account the underlying incidence of the outcome and the variable relative importance of each risk factor from the development to the validation population. This increase in performance though simple recalibration is important in the effort to encourage the updating of models during validation. The statistical effort in recalibrating a model is slight and the final product of a model better fitted to the population in question and increased model performance worth the added step.

Although we aimed to comprehensively review all published papers on development and validation of undiagnosed diabetes risk prediction models, it should be highlighted that we may have missed some published validation studies. However, the overall result would not be expected to differ significantly with the possible inclusion of more model validation studies.

\section{CONCLUSION}

Without recalibration in the validation of a diabetes risk prediction model, the ability of these models to generate an accurate point estimate of an individual's diabetes risk may be inadequate. The importance of generalizability and validation of current models is repeatedly emphasized in literature, however this is fruitless if extra efforts are not taken to fit the model as best as possible to the new setting. Unfortunately, only a relatively small number of validation studies have included recalibration in their methodologies. Additionally, no prevalent diabetes risk prediction models were recalibrated in an attempt to better fit the model to the validation population. An increased focus on the validation, and particularly recalibration, of existent models will improve the generalizability of the models and likely lead to greater application of diabetes risk prediction models in daily clinical practice. The question that remains is, when is a model ruled sufficiently validated and recalibration / updated? Future research should address this question and allow for the determination of how many validation studies, what type of adjustments need to be made and most importantly, what is optimum performance to justify the implementation of the risk prediction model into clinical practice.

\section{ACKNOWLEDGEMENTS}

KLM was supported by a scholarship from the South African National Research Foundation and the Carl \& Emily Fuchs Foundation.

\section{REFERENCES}

[1] Steyerberg EW. Clinical Prediction Models: A practical approach to development, validation, and updating. Gail M, Krickeberg K, Samet J, Tsiati A, Wong W, editors: Springer; 2009. 500 p.

[2] Steyerberg EW, Borsboom GJ, van Houwelingen $\mathrm{HC}$, Eijkemans MJ, Habbema JDF. Validation and updating of predictive logistic regression models: a study on sample size and shrinkage. Statistics in medicine 2004; 23: 2567-86. http://dx.doi.org/10.1002/sim.1844

[3] Janssen K, Moons K, Kalkman C, Grobbee D, Vergouwe Y. Updating methods improved the performance of a clinical prediction model in new patients. J Clin Epidemiol 2008; 61: 76-86.

http://dx.doi.org/10.1016/j.jclinepi.2007.04.018

[4] Collins GS, de Groot JA, Dutton S, Omar O, Shanyinde M, Tajar A, et al. External validation of multivariable prediction models: a systematic review of methodological conduct and reporting. BMC Med Res Methodol 2014; 14: 40.

http://dx.doi.org/10.1186/1471-2288-14-40

[5] Noble D, Mathur R, Dent T, Meads C, Greenhalgh T. Risk models and scores for type 2 diabetes: systematic review. BMJ 2011; 343.

[6] Kengne AP, Beulens JW, Peelen LM, Moons KG, van der Schouw YT, Schulze MB, et al. Non-invasive risk scores for prediction of type 2 diabetes (EPIC-InterAct): a validation of existing models. The Lancet Diabetes \& Endocrinology 2014; 2: $19-29$.

http://dx.doi.org/10.1016/S2213-8587(13)70103-7

[7] Buijsse B, Simmons RK, Griffin SJ, Schulze MB. Risk assessment tools for identifying individuals at risk of 
developing type 2 diabetes. Epidemiologic reviews 2011: $\mathrm{mxq} 019$.

[8] Collins GS, Mallett S, Omar O, Yu L-M. Developing risk prediction models for type 2 diabetes: a systematic review of methodology and reporting. BMC medicine 2011; 9: 103. http://dx.doi.org/10.1186/1741-7015-9-103

[9] Thoopputra T, Newby D, Schneider J, Li SC. Survey of diabetes risk assessment tools: concepts, structure and performance. Diabetes Metab Res Rev 2012; 28: 485-98. http://dx.doi.org/10.1002/dmrr.2296

[10] Brown N, Critchley J, Bogowicz P, Mayige M, Unwin N. Risk scores based on self-reported or available clinical data to detect undiagnosed type 2 diabetes: a systematic review. Diabetes research and clinical practice 2012; 98: 369-85. http://dx.doi.org/10.1016/j.diabres.2012.09.005

[11] Moher D, Liberati A, Tetzlaff J, Altman DG. Preferred reporting items for systematic reviews and meta-analyses: the PRISMA statement. Annals of internal medicine 2009; 151: $264-9$

http://dx.doi.org/10.7326/0003-4819-151-4-200908180$\underline{00135}$

[12] Justice AC, Covinsky KE, Berlin JA. Assessing the generalizability of prognostic information. Annals of internal medicine 1999; 130: 515-24.

http://dx.doi.org/10.7326/0003-4819-130-6-199903160$\underline{00016}$

[13] Wilson PW, Meigs JB, Sullivan L, Fox CS, Nathan DM, D'Agostino RB. Prediction of incident diabetes mellitus in middle-aged adults: the Framingham Offspring Study. Arch Intern Med 2007; 167: 1068-74.

http://dx.doi.org/10.1001/archinte.167.10.1068

[14] Mann DM, Bertoni AG, Shimbo D, Carnethon MR, Chen H, Jenny NS, et al. Comparative Validity of 3 Diabetes Mellitus Risk Prediction Scoring Models in a Multiethnic US Cohort The Multi-Ethnic Study of Atherosclerosis. American journal of epidemiology 2010; 171: 980-8.

http://dx.doi.org/10.1093/aje/kwq030

[15] Lindström J, Tuomilehto J. The Diabetes Risk Score A practical tool to predict type 2 diabetes risk. Diabetes Care 2003; 26: 725-31.

http://dx.doi.org/10.2337/diacare.26.3.725

[16] Saaristo T, Peltonen M, Lindström J, Saarikoski L, Sundvall $\mathrm{J}$, Eriksson JG, et al. Cross-sectional evaluation of the Finnish Diabetes Risk Score: a tool to identify undetected type 2 diabetes, abnormal glucose tolerance and metabolic syndrome. Diabetes and Vascular Disease Research 2005; 2: $67-72$.

http://dx.doi.org/10.3132/dvdr.2005.011

[17] D'Agostino Sr RB, Grundy S, Sullivan LM, Wilson P. Validation of the Framingham coronary heart disease prediction scores: results of a multiple ethnic groups investigation. Jama 2001; 286: 180-7. http://dx.doi.org/10.1001/jama.286.2.180

[18] Hosmer Jr DW, Lemeshow S. Applied logistic regression: John Wiley \& Sons; 2004.

[19] Ivanov J, Tu JV, Naylor CD. Ready-made, recalibrated, or remodeled? Issues in the use of risk indexes for assessing mortality after coronary artery bypass graft surgery. Circulation 1999; 99: 2098-104.

http://dx.doi.org/10.1161/01.CIR.99.16.2098

[20] Toll D, Janssen K, Vergouwe Y, Moons K. Validation, updating and impact of clinical prediction rules: a review. $\mathrm{J}$ Clin Epidemiol 2008; 61: 1085-94.

http://dx.doi.org/10.1016/j.jclinepi.2008.04.008

[21] Nelson W. Applied life data analysis, 1982. Statistics, Published by John Wiley \& Sons, Inc, New York, NY.

[22] Cox D. Regression models and life-tables JR Statist Soc B 34: 187-220. Find this article online 1972.
[23] van Houwelingen HC. Validation, calibration, revision and combination of prognostic survival models. Statistics in medicine 2000; 19: 3401-15.

http://dx.doi.org/10.1002/10970258(20001230)19:24<3401::AID-SIM554>3.0.CO;2-2

[24] Bozorgmanesh M, Hadaegh F, Ghaffari S, Harati H, Azizi F. A simple risk score effectively predicted type 2 diabetes in Iranian adult population: population-based cohort study. The European Journal of Public Health 2011; 21: 554-9. http://dx.doi.org/10.1093/eurpub/ckq074

[25] Hartwig S, Kuss O, Tiller D, Greiser K, Schulze M, Dierkes J et al. Validation of the German Diabetes Risk Score within a population-based representative cohort. Diabetic Medicine 2013; 30: 1047-53

http://dx.doi.org/10.1111/dme.12216

[26] Hippisley-Cox J, Coupland C, Brindle P. The performance of seven QPrediction risk scores in an independent external sample of patients from general practice: a validation study. BMJ Open 2014; 4: e005809.

[27] Rosella LC, Manuel DG, Burchill C, Stukel TA. A populationbased risk algorithm for the development of diabetes: development and validation of the Diabetes Population Risk Tool (DPoRT). J Epidemiol Community Health 2011; 65: 61320

http://dx.doi.org/10.1136/jech.2009.102244

[28] Nichols GA, Brown JB. Validating the Framingham Offspring Study equations for predicting incident diabetes mellitus. Am J Manag Care 2008; 14: 574-80.

[29] Xu L, Jiang C, Schooling C, Zhang W, Cheng K, Lam T. Prediction of 4-year incident diabetes in older Chinese: Recalibration of the Framingham diabetes score on Guangzhou Biobank Cohort Study. Preventive medicine 2014; 69: 63-8.

http://dx.doi.org/10.1016/j.ypmed.2014.09.004

[30] Abbasi A, Corpeleijn E, Peelen LM, Gansevoort RT, de Jong $\mathrm{PE}, \mathrm{Gans} \mathrm{RO}$, et al. External validation of the KORA S4/F4 prediction models for the risk of developing type 2 diabetes in older adults: the PREVEND study. Eur J Epidemiol 2012; 27: 47-52.

http://dx.doi.org/10.1007/s10654-011-9648-4

[31] Rathmann W, Kowall B, Heier M, Herder C, Holle R, Thorand $\mathrm{B}$, et al. Prediction models for incident type 2 diabetes mellitus in the older population: KORA S4/F4 cohort study. Diabetic Medicine 2010; 27: 1116-23. http://dx.doi.org/10.1111/j.1464-5491.2010.03065.x

[32] Abbasi A, Peelen LM, Corpeleijn E, van der Schouw YT, Stolk RP, Spijkerman AM, et al. Prediction models for risk of developing type 2 diabetes: systematic literature search and independent external validation study. BMJ: British Medical Journal 2012; 345.

[33] Alssema M, Vistisen D, Heymans M, Nijpels G, Glümer C, Zimmet $\mathrm{P}$, et al. The Evaluation of Screening and Early Detection Strategies for Type 2 Diabetes and Impaired Glucose Tolerance (DETECT-2) update of the Finnish diabetes risk score for prediction of incident type 2 diabetes. Diabetologia 2011; 54: 1004-12. http://dx.doi.org/10.1007/s00125-010-1990-7

[34] Wannamethee S, Papacosta O, Whincup P, Thomas M Carson C, Lawlor D, et al. The potential for a two-stage diabetes risk algorithm combining non-laboratory-based scores with subsequent routine non-fasting blood tests: results from prospective studies in older men and women. Diabetic Medicine 2011; 28: 23-30. http://dx.doi.org/10.1111/j.1464-5491.2010.03171.x

[35] Chen L, Magliano DJ, Balkau B, Colagiuri S, Zimmet PZ, Tonkin AM, et al. AUSDRISK: an Australian Type 2 Diabetes Risk Assessment Tool based on demographic, lifestyle and simple anthropometric measures. Medical Journal of Australia 2010; 192: 197. 
[36] Joseph J, Svartberg J, Njølstad I, Schirmer H. Incidence of and risk factors for type-2 diabetes in a general population: The Troms $\varnothing$ Study. Scandinavian journal of public health 2010; 38: 768-75.

http://dx.doi.org/10.1177/1403494810380299

[37] Kahn HS, Cheng YJ, Thompson TJ, Imperatore G, Gregg EW. Two risk-scoring systems for predicting incident diabetes mellitus in US adults age 45 to 64 years. Annals of Internal Medicine 2009; 150: 741-51.

http://dx.doi.org/10.7326/0003-4819-150-11-200906020$\underline{00002}$

[38] Hippisley-Cox J, Coupland C, Robson J, Sheikh A, Brindle P. Predicting risk of type 2 diabetes in England and Wales: prospective derivation and validation of QDScore. Bmj 2009; 338.

http://dx.doi.org/10.1136/bmj.b880

[39] Balkau B, Lange C, Fezeu L, Tichet J, de Lauzon-Guillain B, Czernichow S, et al. Predicting Diabetes: Clinical, Biological, and Genetic Approaches Data from the Epidemiological Study on the Insulin Resistance Syndrome (DESIR). Diabetes Care 2008; 31: 2056-61.

http://dx.doi.org/10.2337/dc08-0368

[40] Simmons R, Harding AH, Wareham N, Griffin S. Do simple questions about diet and physical activity help to identify those at risk of Type 2 diabetes? Diabetic Medicine 2007; 24: 830-5.

http://dx.doi.org/10.1111/j.1464-5491.2007.02173.x

[41] Schulze MB, Hoffmann K, Boeing $H$, Linseisen J, Rohrmann $\mathrm{S}$, Möhlig $\mathrm{M}$, et al. An accurate risk score based on anthropometric, dietary, and lifestyle factors to predict the development of type 2 diabetes. Diabetes Care 2007; 30: 510-5. http://dx.doi.org/10.2337/dc06-2089

[42] Stern MP, Williams K, Haffner SM. Identification of persons at high risk for type 2 diabetes mellitus: do we need the oral glucose tolerance test? Annals of Internal Medicine 2002; 136: $575-81$.

http://dx.doi.org/10.7326/0003-4819-136-8-200204160$\underline{00006}$

[43] Von Eckardstein A, Schulte H, Assmann G. Risk for diabetes mellitus in middle-aged Caucasian male participants of the PROCAM study: implications for the definition of impaired fasting glucose by the American Diabetes Association. The Journal of Clinical Endocrinology \& Metabolism 2000; 85: 3101-8.

http://dx.doi.org/10.1210/jcem.85.9.6773

[44] Stern MP, Morales PA, Valdez RA, Monterrosa A, Haffner $\mathrm{SM}$, Mitchell BD, et al. Predicting diabetes: moving beyond impaired glucose tolerance. Diabetes 1993; 42: 706-14. http://dx.doi.org/10.2337/diab.42.5.706

[45] Abdul-Ghani MA, Lyssenko V, Tuomi T, DeFronzo RA, Groop L. Fasting Versus Postload Plasma Glucose Concentration and the Risk for Future Type 2 Diabetes Results from the Botnia Study. Diabetes Care 2009; 32: 2816.

http://dx.doi.org/10.2337/dc08-1264

[46] Kanaya AM, Fyr CLW, De Rekeneire N, Shorr RI, Schwartz $\mathrm{AV}$, Goodpaster $\mathrm{BH}$, et al. Predicting the Development of Diabetes in Older Adults The derivation and validation of a prediction rule. Diabetes Care 2005; 28: 404-8. http://dx.doi.org/10.2337/diacare.28.2.404

[47] Adhikari P, Pathak R, Kotian S. Validation of the MDRFIndian Diabetes Risk Score (IDRS) in another South Indian Population through the Boloor Diabetes Study (BDS). JAPI 2010; 50: 434-6.

[48] Mohan V, Deepa R, Deepa M, Somannavar S, Datta M. A simplified Indian Diabetes Risk Score for screening for undiagnosed diabetic subjects. Journal of the Association of Physicians of India 2005; 53: 759-63.
[49] Akyil RC, Miloglu O, Olgun N, Bayrakdar IS. A comparison of three different diabetes screening methods among dental patients in Turkey. Pakistan journal of medical sciences 2014; 30: 65.

[50] Al Khalaf M, Eid M, Najjar H, Alhajry K, Doi S, Thalib L. Home Eastern Mediterranean Health Journal| Past issues| Volume 16, 2010| Volume 16, issue 7| Screening for diabetes in Kuwait and evaluation of risk scores. EMHJ 2010; 16: 72531.

[51] Herman WH, Smith PJ, Thompson TJ, Engelgau MM, Aubert RE. A new and simple questionnaire to identify people at increased risk for undiagnosed diabetes. Diabetes Care 1995; 18: 382-7.

http://dx.doi.org/10.2337/diacare.18.3.382

[52] Baan CA, Ruige JB, Stolk RP, Witteman J, Dekker JM, Heine $\mathrm{RJ}$, et al. Performance of a predictive model to identify undiagnosed diabetes in a health care setting. Diabetes Care 1999; 22: 213-9.

http://dx.doi.org/10.2337/diacare.22.2.213

[53] Griffin S, Little P, Hales C, Kinmonth A, Wareham N. Diabetes risk score: towards earlier detection of type 2 diabetes in general practice. Diabetes Metab Res Rev 2000; 16: 164-71.

http://dx.doi.org/10.1002/1520-

7560(200005/06)16:3<164::AID-DMRR103>3.0.CO;2-R

[54] Glümer C, Carstensen B, Sandbæk A, Lauritzen T, Jørgensen T, Borch-Johnsen K. A Danish Diabetes Risk Score for Targeted Screening The Inter99 study. Diabetes Care 2004; 27: 727-33.

http://dx.doi.org/10.2337/diacare.27.3.727

[55] Aekplakorn W, Bunnag P, Woodward M, Sritara P, Cheepudomwit S, Yamwong S, et al. A risk score for predicting incident diabetes in the Thai population. Diabetes Care 2006; 29: 1872-7.

http://dx.doi.org/10.2337/dc05-2141

[56] Al-Lawati J, Tuomilehto J. Diabetes risk score in Oman: a tool to identify prevalent type 2 diabetes among Arabs of the Middle East. Diabetes Res Clin Pract 2007; 77: 438-44. http://dx.doi.org/10.1016/j.diabres.2007.01.013

[57] Alssema M, Newson RS, Bakker SJ, Stehouwer CD, Heymans MW, Nijpels G, et al. One risk assessment tool for cardiovascular disease, type 2 diabetes, and chronic kidney disease. Diabetes Care 2012; 35: 741-8. http://dx.doi.org/10.2337/dc11-1417

[58] Bang $\mathrm{H}$, Edwards AM, Bomback AS, Ballantyne CM, Brillon $\mathrm{D}$, Callahan MA, et al. Development and validation of a patient self-assessment score for diabetes risk. Annals of internal medicine 2009; 151: 775-83.

http://dx.doi.org/10.7326/0003-4819-151-11-200912010$\underline{00005}$

[59] Bergmann A, Li J, Wang L, Schulze J, Bornstein S, Schwarz P. A simplified Finnish diabetes risk score to predict type 2 diabetes risk and disease evolution in a German population. Hormone and metabolic research 2007; 39: 677-82. http://dx.doi.org/10.1055/s-2007-985353

[60] Bhadoria AS, Kasar PK, Toppo NA. Validation of Indian Diabetic Risk Score in Diagnosing Type 2 Diabetes Mellitus Against High Fasting Blood Sugar Levels among Adult Population of Central India. Biomedical journal 2014.

[61] Bozorgmanesh M, Hadaegh F, Azizi F. Transportability of the updated diabetes prediction model from Atherosclerosis Risk in Communities Study to a Middle Eastern adult population: community-based cohort study. Acta diabetologica 2013; 50: 175-81.

http://dx.doi.org/10.1007/s00592-010-0241-1

[62] Bozorgmanesh M, Hadaegh F, Zabetian A, Azizi F. San Antonio heart study diabetes prediction model applicable to a Middle Eastern population? Tehran glucose and lipid study. Int J Public Health 2010; 55: 315-23.

http://dx.doi.org/10.1007/s00038-010-0130-y 
[63] Chaturvedi V, Reddy K, Prabhakaran D, Jeemon P, Ramakrishnan L, Shah P, et al. Development of a clinical risk score in predicting undiagnosed diabetes in urban Asian Indian adults: a population-based study. CVD prevention and control 2008; 3: 141-51.

http://dx.doi.org/10.1016/j.cvdpc.2008.07.002

[64] Cameron A, Zimmet P, Soderberg S, Alberti K, Sicree R, Tuomilehto J, et al. The metabolic syndrome as a predictor of incident diabetes mellitus in Mauritius. Diabetic medicine 2007; 24: 1460-9.

http://dx.doi.org/10.1111/j.1464-5491.2007.02288.x

[65] Cameron A, Magliano D, Zimmet P, Welborn T, Colagiuri S, Tonkin A, et al. The metabolic syndrome as a tool for predicting future diabetes: the AusDiab study. J Intern Med 2008; 264: 177-86.

http://dx.doi.org/10.1111/j.1365-2796.2008.01935.x

[66] Chien K, Cai T, Hsu H, Su T, Chang W, Chen M, et al. A prediction model for type 2 diabetes risk among Chinese people. Diabetologia 2009; 52: 443-50.

http://dx.doi.org/10.1007/s00125-008-1232-4

[67] Farran B, Channanath AM, Behbehani K, Thanaraj TA. Predictive models to assess risk of type 2 diabetes, hypertension and comorbidity: machine-learning algorithms and validation using national health data from Kuwait-a cohort study. BMJ Open 2013; 3: e002457.

http://dx.doi.org/10.1136/bmjopen-2012-002457

[68] Franciosi M, De Berardis G, Rossi MC, Sacco M, Belfiglio M, Pellegrini $F$, et al. Use of the diabetes risk score for opportunistic screening of undiagnosed diabetes and impaired glucose tolerance the IGLOO (Impaired Glucose Tolerance and Long-Term Outcomes Observational) study. Diabetes Care 2005; 28: 1187-94.

http://dx.doi.org/10.2337/diacare.28.5.1187

[69] Gao W, Dong Y, Pang Z, Nan H, Wang S, Ren J, et al. A simple Chinese risk score for undiagnosed diabetes. Diabetic Medicine 2010; 27: 274-81.

http://dx.doi.org/10.1111/j.1464-5491.2010.02943.x

[70] Ramachandran A, Snehalatha C, Vijay V, Wareham N, Colagiuri S. Derivation and validation of diabetes risk score for urban Asian Indians. Diabetes Res Clin Pract 2005; 70: 63-70. http://dx.doi.org/10.1016/j.diabres.2005.02.016

[71] Ginde AA, Delaney KE, Lieberman RM, Vanderweil SG, Camargo CA. Estimated risk for undiagnosed diabetes in the emergency department: a multicenter survey. Academic Emergency Medicine 2007; 14: 492-5. http://dx.doi.org/10.1111/j.1553-2712.2007.tb01815.x

[72] Glümer C, Borch-Johnsen K, Colagiuri S. Can a screening programme for diabetes be applied to another population? Diabetic medicine 2005; 22: 1234-8.

http://dx.doi.org/10.1111/j.1464-5491.2005.01641.x

[73] Glümer C, Vistisen D, Borch-Johnsen K, Colagiuri S. Risk scores for type 2 diabetes can be applied in some populations but not all. Diabetes Care 2006; 29: 410-4. http://dx.doi.org/10.2337/diacare.29.02.06.dc05-0945

[74] Gray L, Taub N, Khunti K, Gardiner E, Hiles S, Webb D, et al. The Leicester Risk Assessment score for detecting undiagnosed type 2 diabetes and impaired glucose regulation for use in a multiethnic UK setting. Diabetic Medicine 2010; 27: 887-95.

http://dx.doi.org/10.1111/j.1464-5491.2010.03037.x

[75] Gray L, Davies M, Hiles S, Taub N, Webb D, Srinivasan B, et al. Detection of impaired glucose regulation and/or type 2 diabetes mellitus, using primary care electronic data, in a multiethnic UK community setting. Diabetologia 2012; 55: 959-66.

http://dx.doi.org/10.1007/s00125-011-2432-x

[76] Gray L, Khunti K, Wilmot E, Yates T, Davies M. External validation of two diabetes risk scores in a young UK South Asian population. Diabetes Res Clin Pract 2014; 104: 451-8. http://dx.doi.org/10.1016/j.diabres.2014.03.018
[77] Guasch-Ferré M, Bulló M, Costa B, Martínez-Gonzalez MÁ, Ibarrola-Jurado N, Estruch $\mathrm{R}$, et al. A risk score to predict type 2 diabetes mellitus in an elderly Spanish Mediterranean population at high cardiovascular risk. PLoS One 2012; 7: e33437.

http://dx.doi.org/10.1371/journal.pone.0033437

[78] Guerrero-Romero F, Rodríguez-Morán M. Validation of an instrument for screening cases of type 2 diabetes and monitoring at-risk individuals in Mexico. Revista Panamericana de Salud Pública 2010; 27: 181-6. http://dx.doi.org/10.1590/S1020-49892010000300005

[79] Hanley AJ, Festa A, D'Agostino RB, Wagenknecht LE, Savage PJ, Tracy RP, et al. Metabolic and inflammation variable clusters and prediction of type 2 diabetes factor analysis using directly measured insulin sensitivity. Diabetes 2004; 53: 1773-81. http://dx.doi.org/10.2337/diabetes.53.7.1773

[80] He S, Chen X, Cui K, Peng Y, Liu K, Lv Z, et al. Validity evaluation of recently published diabetes risk scoring models in a general Chinese population. Diabetes Res Clin Pract 2012; 95: 291-8

http://dx.doi.org/10.1016/j.diabres.2011.10.039

[81] Heianza Y, Arase Y, Saito K, Hsieh SD, Tsuji H, Kodama S et al. Development of a screening score for undiagnosed diabetes and its application in estimating absolute risk of future type 2 diabetes in Japan: Toranomon Hospital Health Management Center Study 10 (TOPICS 10). The Journal of Clinical Endocrinology \& Metabolism 2013; 98: 1051-60. http://dx.doi.org/10.1210/jc.2012-3092

[82] Heldgaard PE, Griffin S. Routinely collected general practice data aids identification of people with hyperglycaemia and metabolic syndrome. Diabetic medicine 2006; 23: 996-1002. http://dx.doi.org/10.1111/j.1464-5491.2006.01929.x

[83] Keesukphan P, Chanprasertyothin S, Ongphiphadhanakul B Puavilai G. The development and validation of a diabetes risk score for high-risk Thai adults. Journal-Medical Association of Thailand 2007; 90: 149.

[84] Schmidt MI, Duncan BB, Bang H, Pankow JS, Ballantyne $\mathrm{CM}$, Golden $\mathrm{SH}$, et al. Identifying Individuals at High Risk for Diabetes The Atherosclerosis Risk in Communities study. Diabetes care 2005; 28: 2013-8.

http://dx.doi.org/10.2337/diacare.28.8.2013

[85] Ko G, So W, Tong P, Ma R, Kong A, Ozaki R, et al. A simple risk score to identify Southern Chinese at high risk for diabetes. Diabetic Medicine 2010; 27: 644-9. http://dx.doi.org/10.1111/j.1464-5491.2010.02993.x

[86] Ku G, Kegels G. The performance of the Finnish Diabetes Risk Score, a modified Finnish Diabetes Risk Score and a simplified Finnish Diabetes Risk Score in community-based cross-sectional screening of undiagnosed type 2 diabetes in the Philippines. Primary care diabetes 2013; 7: 249-59. http://dx.doi.org/10.1016/j.pcd.2013.07.004

[87] Lee Y-h, Bang H, Kim HC, Kim HM, Park SW, Kim DJ. A Simple Screening Score for Diabetes for the Korean Population Development, validation, and comparison with other scores. Diabetes Care 2012; 35: 1723-30. http://dx.doi.org/10.2337/dc11-2347

[88] Li J, Bornstein SR, Landgraf R, Schwarz PE. Validation of a simple clinical diabetes prediction model in a middle-aged, white, German population. Arch Intern Med 2007; 167: 25289.

http://dx.doi.org/10.1001/archinte.167.22.2528-c

[89] Li J, Bergmann A, Reimann M, Bornstein S, Schwarz P. A more simplified Finnish diabetes risk score for opportunistic screening of undiagnosed type 2 diabetes in a German population with a family history of the metabolic syndrome. Hormone and metabolic research 2009; 41: 98-103. http://dx.doi.org/10.1055/s-0028-1087191

[90] Li C-I, Chien L, Liu C-S, Lin W-Y, Lai M-M, Lee C-C, et al. Prospective Validation of American Diabetes Association 
Risk Tool for Predicting Pre-Diabetes and Diabetes in Taiwan-Taichung Community Health Study. PLoS One 2011; 6: e25906.

[91] Association AD. Screening for type 2 diabetes. Diabetes Care 2004; 27: S11.

[92] Lin J-W, Chang Y-C, Li H-Y, Chien Y-F, Wu M-Y, Tsai R-Y, et al. Cross-sectional validation of diabetes risk scores for predicting diabetes, metabolic syndrome, and chronic kidney disease in Taiwanese. Diabetes Care 2009; 32: 2294-6. http://dx.doi.org/10.2337/dc09-0694

[93] Luo S, Han L, Zeng P, Chen F, Pan L, Wang S, et al. A Risk Assessment Model for Type 2 Diabetes in Chinese. PLoS One 2014; 9: e104046. http://dx.doi.org/10.1371/journal.pone.0104046

[94] Liu M, Pan C, Jin M. A Chinese diabetes risk score for screening of undiagnosed diabetes and abnormal glucose tolerance. Diabetes technology \& therapeutics 2011; 13: 501-

http://dx.doi.org/10.1089/dia.2010.0106

[95] Lyssenko $V$, Jonsson A, Almgren P, Pulizzi N, Isomaa B, Tuomi $\mathrm{T}$, et al. Clinical risk factors, DNA variants, and the development of type 2 diabetes. New England Journal of Medicine 2008; 359: 2220-32. http://dx.doi.org/10.1056/NEJMoa0801869

[96] Lyssenko V, Jørgensen T, Gerwien RW, Hansen T, Rowe MW, McKenna MP, et al. Validation of a multi-marker model for the prediction of incident type 2 diabetes mellitus: Combined results of the Inter99 and Botnia studies. Diabetes and Vascular Disease Research 2012; 9: 59-67. http://dx.doi.org/10.1177/1479164111424762

[97] Kolberg JA, Jørgensen T, Gerwien RW, Hamren S, McKenna MP, Moler E, et al. Development of a type 2 diabetes risk model from a panel of serum biomarkers from the Inter99 cohort. Diabetes Care 2009; 32: 1207-12. http://dx.doi.org/10.2337/dc08-1935

[98] Mainous AG, Diaz VA, Everett CJ. Assessing risk for development of diabetes in young adults. The Annals of Family Medicine 2007; 5: 425-9.

http://dx.doi.org/10.1370/afm.705

[99] Makrilakis K, Liatis S, Grammatikou S, Perrea D, Stathi C, Tsiligros $P$, et al. Validation of the Finnish diabetes risk score (FINDRISC) questionnaire for screening for undiagnosed type 2 diabetes, dysglycaemia and the metabolic syndrome in Greece. Diabetes Metab 2011; 37: 144-51. http://dx.doi.org/10.1016/i.diabet.2010.09.006

[100] McNeely MJ, Boyko EJ, Leonetti DL, Kahn SE, Fujimoto WY. Comparison of a clinical model, the oral glucose tolerance test, and fasting glucose for prediction of type 2 diabetes risk in Japanese Americans. Diabetes Care 2003; 26: 758-63. http://dx.doi.org/10.2337/diacare.26.3.758

[101] Mühlenbruch K, Ludwig T, Jeppesen C, Joost H-G, Rathmann W, Meisinger C, et al. Update of the German Diabetes Risk Score and external validation in the German MONICA/ KORA study. Diabetes Res Clin Pract 2014; 104: 459-66. http://dx.doi.org/10.1016/..diabres.2014.03.013

[102] Park P, Griffin S, Sargeant L, Wareham N. The performance of a risk score in predicting undiagnosed hyperglycemia. Diabetes Care 2002; 25: 984-8.

http://dx.doi.org/10.2337/diacare.25.6.984

[103] Phillips CM, Kearney PM, McCarthy VJ, Harrington JM, Fitzgerald AP, Perry IJ. Comparison of Diabetes Risk Score Estimates and Cardiometabolic Risk Profiles in a MiddleAged Irish Population. PLoS One 2013; 8: e78950. http://dx.doi.org/10.1371/journal.pone.0078950

[104] Rahman M, Simmons RK, Harding A-H, Wareham NJ, Griffin SJ. A simple risk score identifies individuals at high risk of developing type 2 diabetes: a prospective cohort study. Fam Pract 2008; 25: 191-6.

http://dx.doi.org/10.1093/fampra/cmn024
[105] Rathmann W, Martin S, Haastert B, Icks A, Holle R, Löwel H, et al. Performance of screening questionnaires and risk scores for undiagnosed diabetes: the KORA Survey 2000. Arch Intern Med 2005; 165: 436-41.

http://dx.doi.org/10.1001/archinte.165.4.436

[106] Riaz M, Basit A, Hydrie MZI, Shaheen F, Hussain A, Hakeem $\mathrm{R}$, et al. Risk assessment of Pakistani individuals for diabetes (RAPID). Primary care diabetes 2012; 6: 297-302. http://dx.doi.org/10.1016/j.pcd.2012.04.002

[107] Rolka DB, Narayan KV, Thompson TJ, Goldman D, Lindenmayer J, Alich $\mathrm{K}$, et al. Performance of recommended screening tests for undiagnosed diabetes and dysglycemia. Diabetes Care 2001; 24: 1899-903. http://dx.doi.org/10.2337/diacare.24.11.1899

[108] Rowe MW, Bergman RN, Wagenknecht LE, Kolberg JA. Performance of a multi-marker Diabetes Risk Score in the Insulin Resistance Atherosclerosis Study (IRAS), a multiethnic US cohort. Diabetes Metab Res Rev 2012; 28: 51926. http://dx.doi.org/10.1002/dmrr.2305

[109] Ruige JB, de Neeling JND, Kostense PJ, Bouter LM, Heine RJ. Performance of an NIDDM screening questionnaire based on symptoms and risk factors. Diabetes Care 1997; 20: 491-6. http://dx.doi.org/10.2337/diacare.20.4.491

[110] Schmid R, Vollenweider P, Bastardot F, Waeber G, MarquesVidal $P$. Validation of 7 type 2 diabetes mellitus risk scores in a population-based cohort: CoLaus study. Archives of internal medicine 2012; 172: 188-9. http://dx.doi.org/10.1001/archinte.172.2.188

[111] Schmid R, Vollenweider P, Bastardot F, Vaucher J, Waeber G, Marques-Vidal P. Current genetic data do not improve the prediction of type 2 diabetes mellitus: the CoLaus study. The Journal of Clinical Endocrinology \& Metabolism 2012; 97: E1338-E41. http://dx.doi.org/10.1210/jc.2011-3412

[112] Spijkerman AM, Yuyun MF, Griffin SJ, Dekker JM, Nijpels G, Wareham NJ. The Performance of a Risk Score as a Screening Test for Undiagnosed Hyperglycemia in Ethnic Minority Groups Data from the 1999 Health Survey for England. Diabetes Care 2004; 27: 116-22. http://dx.doi.org/10.2337/diacare.27.1.116

[113] Stern MP, Williams K, González-Villalpando C, Hunt KJ, Haffner SM. Does the metabolic syndrome improve identification of individuals at risk of type 2 diabetes and/or cardiovascular disease? Diabetes Care 2004; 27: 2676-81. http://dx.doi.org/10.2337/diacare.27.11.2676

[114] Stern M, Williams K, Eddy D, Kahn R. Validation of prediction of diabetes by the Archimedes model and comparison with other predicting models. Diabetes Care 2008; 31: 1670-1. http://dx.doi.org/10.2337/dc08-0521

[115] Sun F, Tao Q, Zhan S. An accurate risk score for estimation 5 -year risk of type 2 diabetes based on a health screening population in Taiwan. Diabetes Res Clin Pract 2009; 85: 22834.

http://dx.doi.org/10.1016/j.diabres.2009.05.005

[116] Tabaei BP, Herman WH. A multivariate logistic regression equation to screen for diabetes development and validation. Diabetes Care 2002; 25: 1999-2003. http://dx.doi.org/10.2337/diacare.25.11.1999

[117] Talmud PJ, Hingorani AD, Cooper JA, Marmot MG, Brunner EJ, Kumari $\mathrm{M}$, et al. Utility of genetic and non-genetic risk factors in prediction of type 2 diabetes: Whitehall II prospective cohort study. Bmj 2010; 340. http://dx.doi.org/10.1136/bmj.b4838

[118] Tankova T, Chakarova N, Atanassova I, Dakovska L. Evaluation of the Finnish Diabetes Risk Score as a screening tool for impaired fasting glucose, impaired glucose tolerance and undetected diabetes. Diabetes Res Clin Pract 2011; 92: 46-52. http://dx.doi.org/10.1016/j.diabres.2010.12.020 
[119] Tuomilehto J, Lindström J, Hellmich M, Lehmacher W, Westermeier T, Evers $\mathrm{T}$, et al. Development and validation of a risk-score model for subjects with impaired glucose tolerance for the assessment of the risk of type 2 diabetes mellitus-The STOP-NIDDM risk-score. Diabetes Res Clin Pract 2010; 87: 267-74.

http://dx.doi.org/10.1016/j.diabres.2009.11.011

[120] Urdea M, Kolberg J, Wilber J, Gerwien R, Moler E, Rowe M, et al. Validation of a multimarker model for assessing risk of type 2 diabetes from a five-year prospective study of 6784 Danish people (Inter99). J Diabetes Sci Technol 2009; 3: 748-55.

http://dx.doi.org/10.1177/193229680900300422

[121] Wannamethee SG, Shaper AG, Lennon L, Morris RW. Metabolic syndrome vs Framingham Risk Score for prediction of coronary heart disease, stroke, and type 2 diabetes mellitus. Arch Intern Med 2005; 165: 2644-50. http://dx.doi.org/10.1001/archinte.165.22.2644
[122] Witte D, Shipley M, Marmot M, Brunner E. Performance of existing risk scores in screening for undiagnosed diabetes: an external validation study. Diabetic Medicine 2010; 27: 4653.

http://dx.doi.org/10.1111/j.1464-5491.2009.02891.x

[123] Zhang L, Zhang Z, Zhang Y, Hu G, Chen L. Evaluation of Finnish Diabetes Risk Score in Screening Undiagnosed Diabetes and Prediabetes among US Adults by Gender and Race: NHANES 1999-2010. PLoS One 2014; 9: e97865. http://dx.doi.org/10.1371/journal.pone.0097865

[124] Zhou X, Qiao Q, Ji L, Ning F, Yang W, Weng J, et al. Nonlaboratory-Based Risk Assessment Algorithm for Undiagnosed Type 2 Diabetes Developed on a Nation-Wide Diabetes Survey. Diabetes Care 2013; 36: 3944-52. http://dx.doi.org/10.2337/dc13-0593

(C) 2015 Masconi et al.; Licensee Lifescience Global.

This is an open access article licensed under the terms of the Creative Commons Attribution Non-Commercial License (http://creativecommons.org/licenses/by-nc/3.0/) which permits unrestricted, non-commercial use, distribution and reproduction in any medium, provided the work is properly cited. 